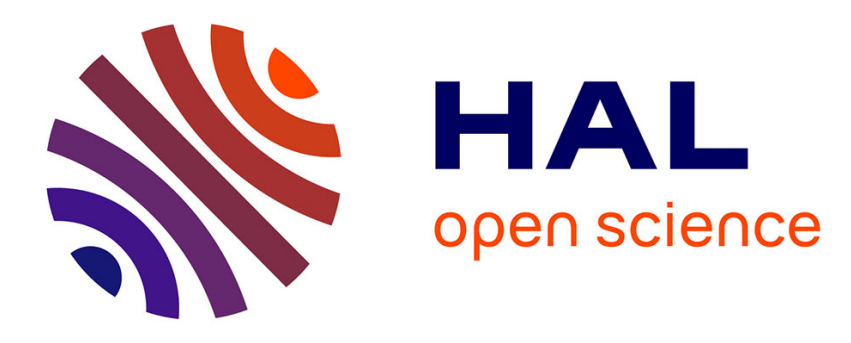

\title{
Design of interval observers for uncertain dynamical systems
}

Denis Efimov, Tarek Raïssi

\section{To cite this version:}

Denis Efimov, Tarek Raïssi. Design of interval observers for uncertain dynamical systems. Automation and Remote Control / Avtomatika i Telemekhanika, 2016, 77 (2), pp.191-225. 10.1134/S0005117916020016 . hal-01276439

\section{HAL Id: hal-01276439 \\ https://inria.hal.science/hal-01276439}

Submitted on 19 Feb 2016

HAL is a multi-disciplinary open access archive for the deposit and dissemination of scientific research documents, whether they are published or not. The documents may come from teaching and research institutions in France or abroad, or from public or private research centers.
L'archive ouverte pluridisciplinaire $\mathbf{H A L}$, est destinée au dépôt et à la diffusion de documents scientifiques de niveau recherche, publiés ou non, émanant des établissements d'enseignement et de recherche français ou étrangers, des laboratoires publics ou privés. 


\title{
Design of interval observers for uncertain dynamical systems
}

Denis Efimov (Non-A project @ Inria, Parc Scientifique de la Haute Borne, 40 avenue Halley, 59650 Villeneuve d'Ascq, France and with Department of Control Systems and Informatics, ITMO University, 49 Kronverkskiy av., 197101 Saint Petersburg, Russia, denis.efimov@inria.fr)

Tarek Raïssi (Conservatoire National des Arts et Métiers (CNAM), Cedric - laetitia 292, Rue St-Martin, 75141 Paris, tarek.raissi@cnam.fr)

\begin{abstract}
Interval state observers provide an estimate on the set of admissible values of the state vector at each instant of time. Ideally, the size of the evaluated set is proportional to the model uncertainty, thus interval observers generate the state estimates with estimation error bounds, similarly to Kalman filters, but in the deterministic framework. Main tools and techniques for design of interval observers are reviewed in this tutorial for continuous-time, discrete-time and time-delayed systems.
\end{abstract}

\section{INTRODUCTION AND MOTIVATION}

The problem of state vector estimation is very challenging and can be encountered in many applications [1], [2], [3]. For linear time-invariant models there are plenty solutions, among them the most popular are Luenberger observer and Kalman filter for deterministic and stochastic settings, respectively. Among other popular solutions for estimations it is worth to mention high-gain observers [4] or high order sliding mode observers/differentiators [5]. In nonlinear case, observer or controller design is based on transformation of the system into a canonical form (frequently close to a linear canonical representation [1], [2], [3]), since such a transformation may depend on uncertain parameters or may be unknown due to the model complexity, then application of the transformation can be an obstruction in practice. See also a nice survey on application of different observers for control and estimation of nonlinear systems [6]. That is why the class of Linear Parameter-Varying (LPV) systems became very popular in applications: a wide class of nonlinear systems can be presented in the LPV form (in this case the system equations are extended). A partial linearity of LPV models allows a rich spectrum of methods developed for linear systems to be applied [7], [8], [9], [10].

Apart of model complexity, another difficulty for an estimator design consists in the model uncertainty (unknown parameters or/and external disturbances). In the presence of uncertainty, design of a conventional estimator, converging to the ideal value of the state, cannot be realized. Application of sliding-mode tools [11] or other disturbance cancellation approaches may resolve this issue in some cases, however, in general, in the presence of uncertainty, the state estimation error is never approaching zero (it can be bounded or asymptotically bounded, and different versions of practical stability are used for analysis). In this case an interval estimation may still remain feasible: an observer can be constructed that, using input-output information, evaluates the set of admissible values (interval) for the state at each instant of time. The interval length has to be minimized by tuning the observer parameters, and it is proportional to the size of the model uncertainty. Despite such a formulation looks like a simplification of the state estimation problem (instead of the state just an interval is estimated), in fact it is an improvement since the interval mean can be used as the state pointwise estimate, while the interval width gives the admissible deviations from that value. Thus, an interval estimator provides a simultaneous accuracy evaluation for bounded uncertainty, which may not have a known statistics (similarly to the Kalman filter, but in the deterministic framework (an Interval Kalman filter has been proposed in [12])).

To illustrate these ideas let us consider a simple example:

$$
\dot{x}(t)=-x^{3}(t)+d(t), t \geq 0,
$$

This work was partially supported by the Government of Russian Federation (Grant 074-U01) and the Ministry of Education and Science of Russian Federation (Project 14.Z50.31.0031). 


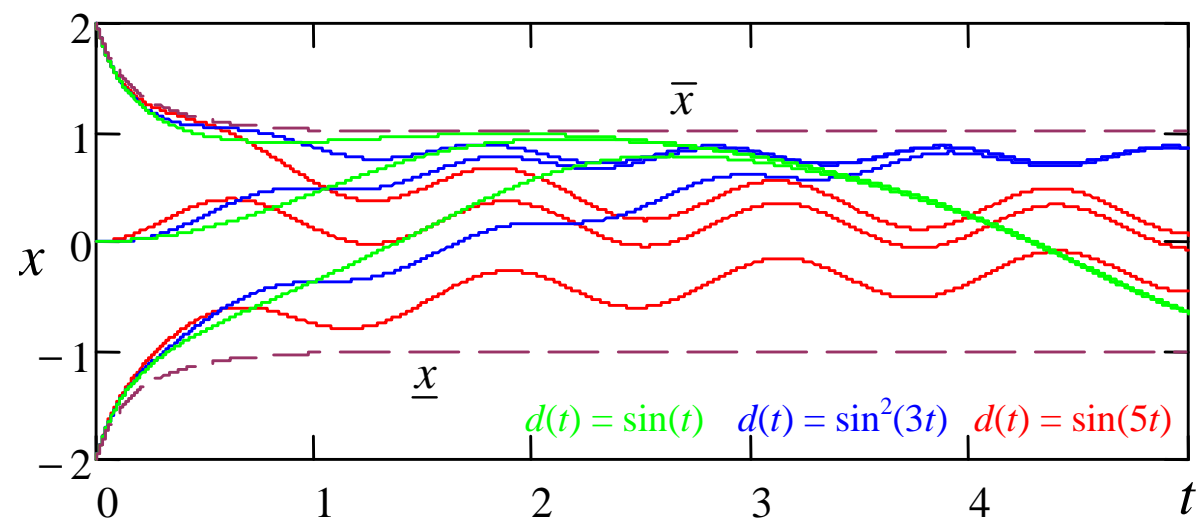

Figure 1. The results of simulation for (1) and (2)

where $x(t)$ is real state variable, $d(t) \in[-1,1]$ is uncertain input whose values belong to the interval $[-1,1]$. We will assume that admissible values for initial conditions of this system belong to the interval $[-2,2]$, i.e. $x(0) \in[-2,2]$. Since the system is nonlinear, the input and initial conditions are uncertain, then it is hard to evaluate an exact value of the state $x(t)$ at each instant of time. However, it is possible to evaluate all admissible values for $x(t)$ with initial conditions $x(0) \in[-2,2]$ and $d(t) \in[-1,1]$. It is possible to show (see the material presented below) that the following system

$$
\begin{gathered}
\underline{\dot{x}}(t)=-\underline{x}^{3}(t)-1, \underline{x}(0)=-2, \\
\dot{\bar{x}}(t)=-\bar{x}^{3}(t)+1, \bar{x}(0)=2
\end{gathered}
$$

has the following property:

$$
\underline{x}(t) \leq x(t) \leq \bar{x}(t) \quad \forall t \geq 0
$$

in other words $x(t) \in[\underline{x}(t), \bar{x}(t)]$ for all $t \geq 0$ and the system (2) estimates an interval of admissible values of $x(t)$, the width of the interval is related to the model (1) uncertainty in $x(0)$ and $d(t)$ (asymptotically only in $d(t)$ ). The results of simulation of these systems for different initial conditions $(0,2$ and -2$)$ and inputs (green lines for $d(t)=\sin (t)$, blue lines for $d(t)=\sin ^{2}(3 t)$ and red lines for $\left.d(t)=\sin (5 t)\right)$ are shown in Fig. 1 together with $\underline{x}(t)$ and $\bar{x}(t)$. As we can conclude, the interval $[\underline{x}(t), \bar{x}(t)]$ represents a worst case estimate of admissible values of $x(t)$ for given uncertainty, and the bounds of the interval are reachable for certain inputs and at some instants of time. The main problem considered in this survey is how to design an interval estimator like (2) using all available information (including the output measurements not presented in (1) due to its simplicity) and minimizing the width of the interval $[\underline{x}(t), \bar{x}(t)]$.

There are several approaches to design interval/set-membership estimators [13], [14], [15], [16], [17]. This survey is devoted to interval observers, which form a subclass of set-membership estimators and whose design is based on the monotone systems theory [15], [17], [18], [19], [20]. This idea has been proposed rather recently in [21], but received already numerous extensions discussed in this paper. In such a way the main restriction for the interval observer design consists in providing cooperativity of the interval estimation error dynamics by a proper design. Such a complexity has been recently overcame in [22], [19], [23] for Linear Time-Invariant (LTI) systems and extended to Linear Time-Varying (LTV), LPV and particular classes of nonlinear systems. In those studies, it has been shown that under some mild conditions, by applying a similarity transformation, a Hurwitz matrix could be transformed to a Hurwitz and Metzler one (cooperative). In the following the main ideas of interval observer design are explained for different classes of systems, in continuous and discrete time and with or without time delays, following the preliminaries, where necessary mathematical notions are introduced.

\section{Preliminaries}

The real and integer numbers are denoted by $\mathbb{R}$ and $\mathbb{Z}$ respectively, $\mathbb{R}_{+}=\{\tau \in \mathbb{R}: \tau \geq 0\}$ and $\mathbb{Z}_{+}=\mathbb{Z} \cap \mathbb{R}_{+}$. Euclidean norm for a vector $x \in \mathbb{R}^{n}$ will be denoted as $|x|$, and for a measurable and locally essentially bounded input $u: \mathbb{R}_{+} \rightarrow \mathbb{R}$ 
$\left(\mathbb{R}_{+}=\{\tau \in \mathbb{R}: \tau \geq 0\}\right)$ the symbol $\|u\|_{\left[t_{0}, t_{1}\right]}$ denotes its $L_{\infty}$ norm:

$$
\|u\|_{\left[t_{0}, t_{1}\right]}=e s s \sup \left\{|u(t)|, t \in\left[t_{0}, t_{1}\right]\right\},
$$

if $t_{1}=+\infty$ then we will simply write $\|u\|$. We will denote as $\mathcal{L}_{\infty}$ the set of all inputs $u$ with the property $\|u\|<\infty$. Denote the sequence of integers $1, \ldots, k$ as $\overline{1, k}$. The symbols $I_{n}, E_{n \times m}$ and $E_{p}$ denote the identity matrix with dimension $n \times n$, the matrix with all elements equal 1 with dimensions $n \times m$ and $p \times 1$, respectively. For a matrix $A \in \mathbb{R}^{n \times n}$ the vector of its eigenvalues is denoted as $\lambda(A), \|\left. A\right|_{\max }=\max _{i=\overline{1, n}, j=\overline{1, n}}\left|A_{i, j}\right|$ (the elementwise maximum norm, it is not submultiplicative) and $\|A\|_{2}=\sqrt{\max _{i=\overline{1, n}} \lambda_{i}\left(A^{\mathrm{T}} A\right)}$ (the induced $L_{2}$ matrix norm), the relation $\|A\|_{\max } \leq\|A\|_{2} \leq n\|A\|_{\max }$ is satisfied between these norms.

\section{A. Interval relations}

For two vectors $x_{1}, x_{2} \in \mathbb{R}^{n}$ or matrices $A_{1}, A_{2} \in \mathbb{R}^{n \times n}$, the relations $x_{1} \leq x_{2}$ and $A_{1} \leq A_{2}$ are understood elementwise. The relation $P \prec 0(P \succ 0)$ means that the matrix $P \in \mathbb{R}^{n \times n}$ is negative (positive) definite. Given a matrix $A \in \mathbb{R}^{m \times n}$, define $A^{+}=\max \{0, A\}, A^{-}=A^{+}-A$ (similarly for vectors) and denote the matrix of absolute values of all elements by $|A|=A^{+}+A^{-}$.

Lemma 1. [20] Let $x \in \mathbb{R}^{n}$ be a vector variable, $\underline{x} \leq x \leq \bar{x}$ for some $\underline{x}, \bar{x} \in \mathbb{R}^{n}$.

(1) If $A \in \mathbb{R}^{m \times n}$ is a constant matrix, then

$$
A^{+} \underline{x}-A^{-} \bar{x} \leq A x \leq A^{+} \bar{x}-A^{-} \underline{x} .
$$

(2) If $A \in \mathbb{R}^{m \times n}$ is a matrix variable and $\underline{A} \leq A \leq \bar{A}$ for some $\underline{A}, \bar{A} \in \mathbb{R}^{m \times n}$, then

$$
\begin{gathered}
\underline{A}^{+} \underline{x}^{+}-\bar{A}^{+} \underline{x}^{-}-\underline{A}^{-} \bar{x}^{+}+\bar{A}^{-} \bar{x}^{-} \leq A x \\
\leq \bar{A}^{+} \bar{x}^{+}-\underline{A}^{+} \bar{x}^{-}-\bar{A} \underline{x}^{+}+\underline{A}^{-} \underline{x}^{-} .
\end{gathered}
$$

Furthermore, if $-\bar{A}=\underline{A} \leq 0 \leq \bar{A}$, then the inequality (4) can be simplified: $-\bar{A}\left(\bar{x}^{+}+\underline{x}^{-}\right) \leq A x \leq \bar{A}\left(\bar{x}^{+}+\underline{x}{ }^{-}\right)$.

\section{B. Nonnegative continuous-time linear systems}

A matrix $A \in \mathbb{R}^{n \times n}$ is called Hurwitz if all its eigenvalues have negative real parts, it is called Metzler if all its elements outside the main diagonal are nonnegative. Any solution of the linear system

$$
\begin{gathered}
\dot{x}=A x+B \omega(t), \omega: \mathbb{R}_{+} \rightarrow \mathbb{R}_{+}^{q}, \omega \in \mathcal{L}_{\infty}^{q}, \\
y=C x+D \omega(t),
\end{gathered}
$$

with $x \in \mathbb{R}^{n}, y \in \mathbb{R}^{p}$ and a Metzler matrix $A \in \mathbb{R}^{n \times n}$, is elementwise nonnegative for all $t \geq 0$ provided that $x(0) \geq 0$ and $B \in \mathbb{R}_{+}^{n \times q}$ [24], [25]. The output solution $y(t)$ is nonnegative if $C \in \mathbb{R}_{+}^{p \times n}$ and $D \in \mathbb{R}_{+}^{p \times q}$. Such dynamical systems are called cooperative (monotone) or nonnegative if only initial conditions in $\mathbb{R}_{+}^{n}$ are considered [24], [25].

For a Metzler matrix $A \in \mathbb{R}^{n \times n}$ its stability can be checked verifying a Linear Programming (LP) problem

$$
A^{\mathrm{T}} \lambda<0
$$

for some $\lambda \in \mathbb{R}_{+}^{n} \backslash\{0\}$, or Lyapunov matrix equation

$$
A^{\mathrm{T}} P+P A \prec 0
$$

for a diagonal matrix $P \in \mathbb{R}^{n \times n}, P>0$ (in general case the matrix $P$ should not be diagonal). The $L_{1}$ and $L_{\infty}$ gains for nonnegative systems (5), i.e. gains of transfer function from input to output in different norms, have been studied in [26], [27], for this kind of systems these gains are interrelated. 
Lemma 2. [26], [27] Let the system (5) be nonnegative (i.e. $A$ is Metzler, $B \geq 0, C \geq 0$ and $D \geq 0$ ), then it is asymptotically stable if and only if there exist $\lambda \in \mathbb{R}_{+}^{n} \backslash\{0\}$ and a scalar $\gamma>0$ such that the following LP problem is feasible:

$$
\left[\begin{array}{c}
A^{T} \lambda+C^{T} E_{p} \\
B^{T} \lambda-\gamma E_{q}+D^{T} E_{p}
\end{array}\right]<0 .
$$

Moreover, in this case $L_{1}$ gain of the operator $\omega \rightarrow y$ is lower than $\gamma$.

Lemma 3. [26], [27] Let the system (5) be cooperative(i.e. $A$ is Metzler, $B \geq 0, C \geq 0$ and $D \geq 0$ ), then it is asymptotically stable if and only if there exist $\lambda \in \mathbb{R}_{+}^{n} \backslash\{0\}$ and a scalar $\gamma>0$ such that the following LP problem is feasible:

$$
\left[\begin{array}{c}
A \lambda+B E_{q} \\
C \lambda-\gamma E_{p}+D E_{q}
\end{array}\right]<0
$$

Moreover, in this case $L_{\infty}$ gain of the transfer $\omega \rightarrow y$ is lower than $\gamma$.

The conventional results and definitions on $L_{2} / L_{\infty}$ stability for linear systems can be found in [28].

\section{Nonnegative discrete-time linear systems}

A matrix $A \in \mathbb{R}^{n \times n}$ is called Schur stable if all its eigenvalues have absolute value less than one, it is called nonnegative if all its elements are nonnegative (i.e. $A \geq 0$ ). Any solution of the system

$$
x_{t+1}=A x_{t}+\omega_{t}, \omega: \mathbb{Z}_{+} \rightarrow \mathbb{R}_{+}^{n}, \omega \in \mathcal{L}_{\infty}^{n}, t \in \mathbb{Z}_{+},
$$

with $x_{t} \in \mathbb{R}^{n}$ and a nonnegative matrix $A \in \mathbb{R}_{+}^{n \times n}$ (for the discrete-time case $\|\omega\|=\sup _{t \in \mathbb{Z}_{+}}\left|\omega_{t}\right|$ and by $\mathcal{L}_{\infty}^{n}$ we denote the set of inputs $\omega: \mathbb{Z}_{+} \rightarrow \mathbb{R}^{n}$ with the property $\left.\|\omega\|<+\infty\right)$, is elementwise nonnegative for all $t \geq 0$ provided that $x(0) \geq 0$ [29]. Such a system is called cooperative (monotone) or nonnegative [29].

Lemma 4. [24] A matrix $A \in \mathbb{R}_{+}^{n \times n}$ is Schur stable iff there exists a diagonal matrix $P \in \mathbb{R}^{n \times n}, P>0$ such that $A^{T} P A-P \prec 0$.

\section{Nonnegative linear systems with delays}

Consider a linear system with constant delays

$$
\dot{x}(t)=A_{0} x(t)+\sum_{i=1}^{N} A_{i} x\left(t-\tau_{i}\right)+\omega(t),
$$

where $x(t) \in \mathbb{R}^{n}$ is the state, $x_{t} \in \mathcal{C}_{\tau}^{n}$ for $\tau=\max _{1 \leq i \leq N} \tau_{i}$ where $\tau_{i} \in \mathbb{R}_{+}$are the delays $\left(\mathcal{C}_{\tau}=C([-\tau, 0], \mathbb{R})\right.$ is the set of continuous maps from $[-\tau, 0]$ into $\mathbb{R}, \mathcal{C}_{\tau+}=\left\{y \in \mathcal{C}_{\tau}: y(s) \in \mathbb{R}_{+}, s \in[-\tau, 0]\right\}$ ); a piecewise continuous function $\omega \in \mathcal{L}_{\infty}^{n}$ is the input; the constant matrices $A_{i}, i=\overline{0, N}$ have appropriate dimensions. The system (6) is called cooperative or nonnegative [30] if it admits $x(t) \in \mathbb{R}_{+}^{n}$ for all $t \geq t_{0}$ provided that $x_{t_{0}} \in \mathcal{C}_{\tau+}^{n}$ and $\omega: \mathbb{R} \rightarrow \mathbb{R}_{+}^{n}$.

Lemma 5. [31], [30] The system (6) is nonnegative for all $\tau \in \mathbb{R}_{+}$iff $A_{0}$ is Metzler and $A_{i}, i=\overline{1, N}$ are nonnegative matrices.

Lemma 6. [31], [30] A nonnegative system (6) is asymptotically stable for $\omega(t) \equiv 0$ for all $\tau \in \mathbb{R}_{+}$iff there are $p, q \in \mathbb{R}_{+}^{n}(p>0$ and $q>0)$ such that

$$
p^{T} \sum_{i=0}^{N} A_{i}+q^{T}=0
$$

Under conditions of the above lemma the system has bounded solutions for $\omega \in \mathcal{L}_{\infty}^{n}$ with $\omega(t) \in \mathbb{R}_{+}^{n}$ for all $t \in \mathbb{R}$.

The delay-independent nonnegativity and stability conditions introduced in lemmas 5 and 6 are general and simple, but rather restrictive. The delay-dependent conditions presented below may enlarge the class of systems under consideration in many application-important situations. 
Lemma 7. [32] The system (6) for $N=1$ with $\omega(t) \in \mathbb{R}_{+}^{n}$ for all $t \in \mathbb{R}$ and initial conditions

$$
x(\theta)=0 \text { for }-\tau \leq \theta<0, x(0) \in \mathbb{R}_{+}^{n},
$$

is nonnegative if $A_{1}$ is Metzler, $A_{0} \geq 0$, and

$$
0 \leq\left(A_{0}\right)_{i, i} \leq-e\left(A_{1}\right)_{i, i}<\left(A_{0}\right)_{i, i}+\tau^{-1}
$$

for all $i=1, \ldots, n, e=\exp (1)$.

\section{INTERVAL OBSERVERS FOR LTI SYSTEMS}

Let us start the line of design of interval observers with the simplest cases of time-invariant linear models in continuous and discrete time.

\section{A. Continuous-time case}

Consider the following system

$$
\dot{x}(t)=A x(t)+d(t), y(t)=C x(t)+v(t), t \in \mathbb{R}_{+}
$$

where $x(t) \in \mathbb{R}^{n}$ is the state, $y(t) \in \mathbb{R}^{p}$ is the output; $d(t) \in \mathbb{R}^{n}$ is the disturbance, $d \in \mathcal{L}_{\infty}^{n} ; v(t) \in \mathbb{R}^{p}$ is the measurement noise, $v \in \mathcal{L}_{\infty}^{p}$; the matrices $A, C$ have appropriate dimensions. This model has three sources of uncertainty: initial conditions for $x(0)$, instant values of $d$ and $v$. It is assumed that all these uncertain factors belong to known intervals.

Assumption 1. Let $x(0) \in\left[\underline{x}_{0}, \bar{x}_{0}\right]$ for some known $\underline{x}_{0}, \bar{x}_{0} \in \mathbb{R}^{n}$, let also two functions $\underline{d}, \bar{d} \in \mathcal{L}_{\infty}^{n}$ and a constant $V>0$ be given such that

$$
\underline{d}(t) \leq d(t) \leq \bar{d}(t),|v(t)| \leq V \quad \forall t \geq 0 .
$$

Thus, by this assumption three intervals $\left[\underline{x}_{0}, \bar{x}_{0}\right],[\underline{d}(t), \bar{d}(t)]$ and $[-V, V]$ determine uncertainty of $x(0), d(t)$ and $v(t)$ respectively. It is required to calculate two estimates $\underline{x}, \bar{x} \in \mathcal{L}_{\infty}^{n}$, using the available information on these intervals and $y(t)$, such that

$$
\underline{x}(t) \leq x(t) \leq \bar{x}(t) \quad \forall t \geq 0
$$

Interval observer is a solution to this problem:

$$
\begin{aligned}
\underline{\dot{x}}(t)= & A \underline{x}(t)+L[y(t)-C \underline{x}(t)]-|L| E_{p} V+\underline{d}(t), \\
\dot{\bar{x}}(t)= & A \bar{x}(t)+L[y(t)-C \bar{x}(t)]+|L| E_{p} V+\bar{d}(t), \\
& \underline{x}(0)=\underline{x}_{0}, \bar{x}(0)=\bar{x}_{0},
\end{aligned}
$$

where $L \in \mathbb{R}^{n \times p}$ is the observer gain to be designed. The conditions to satisfy for $L$ are given below.

Theorem 1. [21] Let Assumption 1 hold and $x \in \mathcal{L}_{\infty}^{n}$, then in the system (7) with the interval observer (9) the relations (8) are satisfied provided that the matrix $A-L C$ is Metzler. In addition, $\underline{x}, \bar{x} \in \mathcal{L}_{\infty}^{n}$ if $A-L C$ is Hurwitz.

To understand the main ideas behind this result (that is very representative for all kinds of interval observers) consider its proof.

Proof. Define two estimation errors

$$
\underline{e}(t)=x(t)-\underline{x}(t), \bar{e}(t)=\bar{x}(t)-x(t),
$$

which yield differential equations:

$$
\begin{aligned}
& \underline{\dot{e}}(t)=[A-L C] \underline{e}(t)+L v(t)+|L| E_{p} V+d(t)-\underline{d}(t), \\
& \dot{\bar{e}}(t)=[A-L C] \bar{e}(t)-L v(t)+|L| E_{p} V+\bar{d}(t)-d(t) .
\end{aligned}
$$


By Assumption 1, for all $t \geq 0$

$$
|L| E_{p} V \pm L v(t) \geq 0, d(t)-\underline{d}(t) \geq 0, \bar{d}(t)-d(t) \geq 0
$$

If $A-L C$ is a Metzler matrix, since all inputs of $\underline{e}(t), \bar{e}(t)$ are positive and $\underline{e}(0) \geq 0, \bar{e}(t) \geq 0$, then $\underline{e}(t) \geq 0, \bar{e}(t) \geq 0$ for all $t \geq 0$ [24], [25]. The property (8) follows from these relations. If $A-L C$ is Hurwitz, since all inputs of $\underline{e}(t), \bar{e}(t)$ are bounded, then $\underline{e}, \bar{e} \in \mathcal{L}_{\infty}^{n}$ and boundedness of $\underline{x}, \bar{x}$ is implied by boundedness of $x$.

Thus, from this proof we can conclude that the idea for design of an interval observer is to guarantee nonnegativity of the estimation error dynamics. The observer gain $L$ has to be designed in a way that the matrix $A-L C$ would be Metzler and Hurwitz. In addition, in order to optimize the width of estimated interval $[\underline{x}(t), \bar{x}(t)]$ the problem of $L_{1}$ or $L_{\infty}$ optimization of that gain value can be posed. Using lemmas 2 and 3 this problem can be formulated as a LP computational procedure (see also [33] for another LP problem formulation and solution for a more generic LPV system), for example, for $L_{1}$ optimization it is necessary to find $\lambda \in \mathbb{R}^{n}, w \in \mathbb{R}^{p}$ and a diagonal matrix $M \in \mathbb{R}^{n \times n}$ such that

$$
\begin{gathered}
{\left[\begin{array}{c}
A^{\mathrm{T}} \lambda-C^{\mathrm{T}} w+E_{n} \\
\lambda-\gamma E_{n}
\end{array}\right]<0,} \\
\lambda>0, M \geq 0, \\
A^{\mathrm{T}} \lambda-C^{\mathrm{T}} w+M \lambda \geq 0,
\end{gathered}
$$

then $w=L^{\mathrm{T}} \lambda[34]$.

Formulation (10) provides an effective computational tool to design interval observers, but it is only sufficient condition and in some cases this LP problem may have no solution, but it does not imply that it is not possible to design an interval observer. Roughly speaking in this case it is not possible to find $L$ such that $A-L C$ is simultaneously Metzler and Hurwitz. It is well known fact that Hurwitz property of a matrix is preserved under a similarity transformation of coordinates, then to overcome the issue it is possible to design the gain $L$ such that the matrix $A-L C$ is Hurwitz and next to find a nonsingular matrix $S \in \mathbb{R}^{n \times n}$ such that in the new coordinates $z=S x$ the state matrix $D=S(A-L C) S^{-1}$ is Metzler (it is Hurwitz by construction). The conditions of existence of such a real transformation matrix $S$ are given in the following lemma.

Lemma 8. [19] Given the matrices $A \in \mathbb{R}^{n \times n}, D \in \mathbb{R}^{n \times n}$ and $C \in \mathbb{R}^{p \times n}$. If there is a matrix $L \in \mathbb{R}^{n \times p}$ such that the matrices $A-L C$ and $D$ have the same eigenvalues, then there is a matrix $S \in \mathbb{R}^{n \times n}$ such that $D=S(A-L C) S^{-1}$ provided that the pairs $\left(A-L C, \chi_{1}\right)$ and $\left(D, \chi_{2}\right)$ are observable for some $\chi_{1} \in \mathbb{R}^{1 \times n}, \chi_{2} \in \mathbb{R}^{1 \times n}$.

Note that (7) can be rewritten as follows:

$$
\dot{x}(t)=(A-L C) x(t)+L y(t)-L v(t)+d(t) .
$$

Under conditions of this lemma and in the new coordinates $z=S x$ the system (7) takes the form:

$$
\dot{z}(t)=D z(t)+S L y(t)+\delta(t), \delta(t)=S[d(t)-L v(t)]
$$

And using Lemma 1 we obtain that

$$
\underline{\delta}(t) \leq \delta(t) \leq \bar{\delta}(t)
$$

where $\underline{\delta}(t)=S^{+} \underline{d}(t)-S^{-} \bar{d}(t)-|S L| E_{p} V$ and $\bar{\delta}(t)=S^{+} \bar{d}(t)-S^{-} \underline{d}(t)+|S L| E_{p} V$. Next, for the system (11) all conditions 
of Theorem 1 are satisfied and an interval observer similar to (9) can be designed:

$$
\begin{gathered}
\underline{\dot{z}}(t)=D \underline{z}(t)+S L y(t)+\underline{\delta}(t), \\
\dot{\bar{z}}(t)=D \bar{z}(t)+S L y(t)+\bar{\delta}(t), \\
\underline{z}(0)=S^{+} \underline{x}_{0}-S^{-} \bar{x}_{0}, \bar{z}(0)=S^{+} \bar{x}_{0}-S^{-} \underline{x}_{0}, \\
\underline{x}(t)=\left(S^{-1}\right)^{+} \underline{z}(t)-\left(S^{-1}\right)^{-} \bar{z}(t), \\
\bar{x}(t)=\left(S^{-1}\right)^{+} \bar{z}(t)-\left(S^{-1}\right)^{-} \underline{z}(t),
\end{gathered}
$$

where the relations (3) are used to calculate the initial conditions for $\underline{z}, \bar{z}$ and the estimates $\underline{x}, \bar{x}$. It is easy to show that in (12) the inclusion (8) is satisfied and $\underline{x}, \bar{x} \in \mathcal{L}_{\infty}^{n}$.

If conditions of Lemma 8 are not satisfied, then it is possible also to ask that transformation to new coordinates be timevarying as in the next lemma.

Lemma 9. [22] Let $A-L C$ be Hurwitz, then there exists an invertible matrix function $P: \mathbb{R} \rightarrow \mathbb{R}^{n \times n}$, of class $C^{\infty}$ elementwise, $\|P(t)\|_{2}<+\infty$ for all $t \in \mathbb{R}$, such that for all $t \in \mathbb{R}$

$$
\dot{P}(t)=D P(t)-P(t)(A-L C)
$$

where $D \in \mathbb{R}^{n \times n}$ is a Hurwitz and Metzler matrix.

A variant of Lemma 9 has been proposed in [23], where complex-valued formulation of the system is used that leads to a more compact design of the transformation matrix.

Under conditions of Lemma 9 in the new coordinates $\zeta(t)=P(t) x(t)$ we obtain

$$
\dot{\zeta}(t)=D \zeta(t)+P(t) L y(t)+\delta(t), \delta(t)=P(t)[d(t)-L v(t)]
$$

and again

$$
\begin{gathered}
\underline{\delta}(t) \leq \delta(t) \leq \bar{\delta}(t), \\
\underline{\delta}(t)=P^{+}(t) \underline{d}(t)-P^{-}(t) \bar{d}(t)-|P(t) L| E_{p} V, \\
\bar{\delta}(t)=P^{+}(t) \bar{d}(t)-P^{-}(t) \underline{d}(t)+|P(t) L| E_{p} V .
\end{gathered}
$$

The interval observer has a form similar to (12) [22]:

$$
\begin{gathered}
\underline{\dot{z}}(t)=D \underline{z}(t)+P(t) L y(t)+\underline{\delta}(t), \\
\dot{\bar{z}}(t)=D \bar{z}(t)+P(t) L y(t)+\bar{\delta}(t), \\
\underline{z}(0)=P^{+}(t) \underline{x}_{0}-P^{-}(t) \bar{x}_{0}, \bar{z}(0)=P^{+}(t) \bar{x}_{0}-P^{-}(t) \underline{x}_{0}, \\
\underline{x}(t)=\left(P^{-1}\right)^{+}(t) \underline{z}(t)-\left(P^{-1}\right)^{-}(t) \bar{z}(t), \\
\bar{x}(t)=\left(P^{-1}\right)^{+}(t) \bar{z}(t)-\left(P^{-1}\right)^{-}(t) \underline{z}(t),
\end{gathered}
$$

however, its realization needs more computations than for (12) due to time-varying transformation.

It is worth to mention that in general any system may be embedded in its internal positive representation [35], which has dimension $2 n$ (then interval observer has dimension $4 n$ ). For example, a matrix can be decomposed as a difference of Metzler and nonnegative matrices:

$$
\begin{aligned}
A-L C & =(A-L C)_{\backslash}+(A-L C)_{\times} \\
& =(A-L C)_{\searrow}+(A-L C)_{\times}^{+}-(A-L C)_{\times}^{-}
\end{aligned}
$$

where $(A-L C) \backslash$ is the diagonal matrix composed by elements of $(A-L C)$ on the main diagonal and $(A-L C)_{\times}=$ 
$A-L C-(A-L C) \backslash$. Then the following interval observer can be proposed for (7):

$$
\begin{aligned}
\underline{\dot{x}}(t)= & {\left[(A-L C) \backslash+(A-L C)_{\times}^{+}\right] \underline{x}(t)-(A-L C)_{\times}^{-} \bar{x}(t) } \\
& +L y(t)-|L| E_{p} V+\underline{d}(t), \\
\dot{\bar{x}}(t)= & {\left[(A-L C) \backslash+(A-L C)_{\times}^{+}\right] \bar{x}(t)-(A-L C)_{\times}^{-} \underline{x}(t) } \\
& +L y(t)+|L| E_{p} V+\bar{d}(t), \\
& \underline{x}(0)=\underline{x}_{0}, \bar{x}(0)=\bar{x}_{0} .
\end{aligned}
$$

Theorem 2. [36] Let Assumption 1 hold and $x \in \mathcal{L}_{\infty}^{n}$, then in the system (7) with the interval observer (13) the relations (8) are satisfied. In addition, $\underline{x}, \bar{x} \in \mathcal{L}_{\infty}^{n}$ if

$$
R=\left[\begin{array}{cc}
A-L C)_{\backslash}+(A-L C)_{\times}^{+} & (A-L C)_{\times}^{-} \\
(A-L C)_{\times}^{-} & A-L C)_{\backslash}+(A-L C)_{\times}^{+}
\end{array}\right]
$$

is Hurwitz.

Proof. Consider again the estimation errors

$$
\begin{gathered}
e(t)=\left[\underline{e}^{\mathrm{T}}(t) \bar{e}^{\mathrm{T}}(t)\right]^{\mathrm{T}}, \\
\underline{e}(t)=x(t)-\underline{x}(t), \bar{e}(t)=\bar{x}(t)-x(t),
\end{gathered}
$$

which yield for (13) differential equations:

$$
\dot{e}(t)=R e(t)+\left[\begin{array}{c}
|L| E_{p} V+L v(t)+d(t)+\underline{d}(t) \\
|L| E_{p} V-L v(t)+\bar{d}(t)-d(t)
\end{array}\right] .
$$

The relations (8) immediately follows under Assumption 1 since $R$ is Metzler (the rest of the proof repeats the case of Theorem 1).

As we can conclude the requirement on Metzler property of the matrix $A-L C$ is completely avoided, and the main difficulty in application of the last theorem consists in finding conditions under which the Metzler matrix $R$ is Hurwitz. It is also difficult to formulate some LMIs to find $L$ in this setting.

By this, the main approaches to design interval observers for LTI systems are presented. If it is possible to find $L$ such that $A-L C$ is Hurwitz and Metzler (looking for a solution of LP problem (10)), then the interval observer (9) is a solution. If such a gain $L$ does not exist, then different transformations of coordinates from lemmas 8 and 9 can be tested. In this case $L$ also can be found as a solution of LP problem or LMIs, and the interval observer (12) can be applied. An alternative solution (13) also can be tested.

Remark 1. Note that developed observers (9), (12) can be easily applied for some nonlinear systems, where all nonlinear terms are contained in $d(t)=d(t, u(t), x(t))$ in a manner that Assumption 1 is satisfied for some $\underline{d}, \bar{d} \in \mathcal{L}_{\infty}^{n}(\underline{d}(t)=\underline{d}(t, u(t), y(t), V)$ and $\bar{d}(t)=\bar{d}(t, u(t), y(t), V))$.

Example 1. Consider an uncertain nonlinear system described by [19]:

$$
\dot{x}=A x+B\left(p_{1}, p_{2}\right) f(x) u(t), y=C x,
$$

where $x \in \mathbb{R}^{3}$ is the state, $f(x)=x_{1} x_{2}$ is a nonlinear function, $u \in \mathbb{R}_{+}$is a positive control input, $\underline{p}_{i} \leq p_{i} \leq \bar{p}_{i}, i=1,2$ are some uncertain parameters,

$$
\begin{gathered}
A=\left[\begin{array}{ccc}
2 & 0 & 0 \\
1 & -4 & \sqrt{3} \\
-1 & -\sqrt{3} & -4
\end{array}\right], B\left(p_{1}, p_{2}\right)=\left[\begin{array}{c}
-2 p_{1} \\
0 \\
p_{2}
\end{array}\right], \\
C=\left[\begin{array}{lll}
1 & 0 & 0
\end{array}\right],
\end{gathered}
$$


with $\underline{p}_{1}=4.48, \bar{p}_{1}=6.12, \underline{p}_{2}=3.2, \bar{p}_{2}=3.6, u(t)=1+\sin (2 t)$. This system has bounded solutions. The pair $(A, C)$ is not observable and there exists no gain $L$ such that the matrix $A-L C$ is Metzler, which makes the techniques of Theorem 1 inappropriate. For the observer gain $L=\left[\begin{array}{ccc}3 & 0 & 0\end{array}\right]^{T}$ the matrix $A-L C$ is Hurwitz and has the eigenvalues $-1,-4 \pm \sqrt{3} i$. Only the first real eigenvalue can be assigned by the gain $L$. The matrix $A-L C$ is not Metzler for any $L$. Thus, to find a transformation of coordinates, it is necessary to construct a Metzler and Hurwitz matrix $D$ with the eigenvalues of $A-L C$. The matrix

$$
D=\left[\begin{array}{ccc}
-a & b & 0 \\
0 & -a & b \\
b & 0 & -a
\end{array}\right]
$$

has eigenvalues $b-a,-a-0.5 b \pm 0.5 b \sqrt{3} i$, therefore in our example we have to choose $b=2$ and $a=3$. The pairs $\left(A-L C, e_{1}\right)$ and $\left(D, e_{2}\right)$ are observable for

$$
e_{1}=\left[\begin{array}{lll}
1 & 0 & 1
\end{array}\right], e_{2}=\left[\begin{array}{lll}
1 & 1 & 0
\end{array}\right]
$$

then

$$
S=\left[\begin{array}{ccc}
0.158 & 0.866 & 0.5 \\
0.842 & -0.866 & 0.5 \\
0.658 & 0 & -1
\end{array}\right]
$$

\section{B. Discrete-time case}

Design of interval observers in discrete time follows approximately the same steps as in the continuous time taking into account the results of subsection II-C on nonnegative systems of this kind. Consider the following system

$$
x_{t+1}=A x_{t}+d_{t}, y_{t}=C x_{t}+v_{t}, t \in \mathbb{Z}_{+}
$$

where $x_{t} \in \mathbb{R}^{n}$ and $y_{t} \in \mathbb{R}^{p}$ are the state and the output as before; $d_{t} \in \mathbb{R}^{n}$ is the disturbance, $d \in \mathcal{L}_{\infty}^{n} ; v_{t} \in \mathbb{R}^{p}$ is the measurement noise, $v \in \mathcal{L}_{\infty}^{p}$; the matrices $A, C$ have appropriate dimensions. This model has also three sources of uncertainty: initial conditions for $x_{0}$, instant values of $d_{t}$ and $v_{t}$, all of them belonging to known intervals.

Assumption 2. Let $x(0) \in\left[\underline{X}_{0}, \bar{X}_{0}\right]$ for some known $\underline{X}_{0}, \bar{X}_{0} \in \mathbb{R}^{n}$, let also two functions $\underline{d}, \bar{d} \in \mathcal{L}_{\infty}^{n}$ and a constant $V>0$ are given such that

$$
\underline{d}_{t} \leq d_{t} \leq \bar{d}_{t},\left|v_{t}\right| \leq V \quad \forall t \in \mathbb{Z}_{+}
$$

It is required to calculate two estimates $\underline{x}, \bar{x} \in \mathcal{L}_{\infty}^{n}$, using the available information on these intervals and $y(t)$, such that

$$
\underline{x}_{t} \leq x_{t} \leq \bar{x}_{t} \quad \forall t \in \mathbb{Z}_{+}
$$

The following simplest interval observer is a solution to this problem:

$$
\begin{aligned}
\underline{x}_{t+1}= & A \underline{x}_{t}+L\left[y_{t}-C \underline{x}_{t}\right]-|L| E_{p} V+\underline{d}_{t}, \\
\bar{x}_{t+1}= & A \bar{x}_{t}+L\left[y_{t}-C \bar{x}_{t}\right]+|L| E_{p} V+\bar{d}_{t}, \\
& \underline{x}_{0}=\underline{X}_{0}, \bar{x}_{0}=\bar{X}_{0},
\end{aligned}
$$

where $L \in \mathbb{R}^{n \times p}$ is the observer gain to be designed as it is required below.

Theorem 3. [37] Let Assumption 2 hold and $x \in \mathcal{L}_{\infty}^{n}$, then in the system (14) with the interval observer (16) the relations (15) are satisfied provided that the matrix $A-L C$ is nonnegative. In addition, $\underline{x}, \bar{x} \in \mathcal{L}_{\infty}^{n}$ if $A-L C$ is Schur stable.

Proof. The system (14) can be equivalently rewritten as follows:

$$
x_{t+1}=(A-L C) x_{t}+L y_{t}-L v_{t}+d_{t} .
$$


Let us again consider behavior of two estimation errors $\underline{e}_{t}=x_{t}-\underline{x}_{t}$ and $\bar{e}_{t}=\bar{x}_{t}-x_{t}$ :

$$
\begin{aligned}
& \underline{e}_{t+1}=(A-L C) \underline{e}_{t}+d_{t}-\underline{d}_{t}+|L| E_{p} V-L v_{t} \\
& \bar{e}_{t+1}=(A-L C) \bar{e}_{t}+\bar{d}_{t}-d_{t}+|L| E_{p} V+L v_{t}
\end{aligned}
$$

By Assumption 2, $d_{t}-\underline{d}_{t}+|L| E_{p} V-L v_{t} \geq 0$ and $\bar{d}_{t}-d_{t}+|L| E_{p} V+L v_{t} \geq 0$ for all $t \in \mathbb{Z}_{+}$, then since $A-L C \geq 0$ and $\underline{e}_{0} \geq 0, \bar{e}_{0} \geq 0$ we conclude that $\underline{e}_{t} \geq 0, \bar{e}_{t} \geq 0$ for all $t \in \mathbb{Z}_{+}$. Therefore, the interval inclusion (15) is satisfied. For a Schur stable matrix $A-L C$ and bounded inputs $L y_{t}-|L| E_{p} V+\underline{d}_{t}, L y_{t}+|L| E_{p} V+\bar{d}_{t}$ the boundedness of $\underline{x}_{t}, \bar{x}_{t}$ immediately follows by standard arguments.

The matrix $L$ can be found as a solution of the following LMIs [37]:

$$
\begin{gathered}
{\left[\begin{array}{cc}
P & P A-W C \\
A^{\mathrm{T}} P-C^{\mathrm{T}} W^{\mathrm{T}} & P
\end{array}\right] \succ 0, P \succ 0,} \\
P A-W C \geq 0
\end{gathered}
$$

where $P \in \mathbb{R}^{n \times n}$ is diagonal and $W \in \mathbb{R}^{n \times p}$ are two matrix variables to determine, then $L=P^{-1} W$. A gain optimization problem can also be formulated to find $L$ providing a minimal interval width $\bar{x}_{t}-\underline{x}_{t}$ in some sense for given in Assumption 2 uncertainty.

If it is not possible to find a solution of (17), then the interval observer can be constructed in new coordinates using constant or time-varying state transformation [38], [37], [39] (Lemma 8 can be directly applied in the discrete-time case).

Theorem 4. [37] Let Assumption 2 be satisfied, the matrix $A-L C$ is Schur stable and $x \in \mathcal{L}_{\infty}^{n}$ in (14), there exists matrix $R \in \mathbb{R}_{+}^{n \times n}$ such that $\lambda(A-L C)=\lambda(R)$ and the pairs $\left(A-L C, e_{1}\right),\left(R, e_{2}\right)$ are observable for some $e_{1} \in \mathbb{R}^{1 \times n}, e_{2} \in \mathbb{R}^{1 \times n}$. Then for all $t \in \mathbb{Z}_{+}$the estimates $\underline{x}_{t}, \bar{x}_{t}$ are bounded and the relations (15) are satisfied for

$$
\begin{aligned}
& \underline{x}_{t}=S^{+} \underline{z}_{t}-S^{-} \bar{z}_{t}, \bar{x}_{t}=S^{+} \bar{z}_{t}-S^{-} \underline{z}_{t} \\
& \underline{z}_{t+1}=R \underline{z}_{t}+F y_{t}-|F| E_{p} V \\
& +\left(S^{-1}\right)^{+} \underline{d}_{t}-\left(S^{-1}\right)^{-} \bar{d}_{t}, \\
& \bar{z}_{t+1}=R \bar{z}_{t}+F y_{t}+|F| E_{p} V \\
& +\left(S^{-1}\right)^{+} \bar{d}_{t}-\left(S^{-1}\right)^{-} \underline{d}_{t} ; \\
& \underline{z}_{0}=\left(S^{-1}\right)^{+} \underline{X}_{0}-\left(S^{-1}\right)^{-} \bar{X}_{0}, \\
& \bar{z}_{0}=\left(S^{-1}\right)^{+} \bar{X}_{0}-\left(S^{-1}\right)^{-} \underline{X}_{0},
\end{aligned}
$$

where $S=O_{R} O_{A-L C}^{-1}\left(O_{A-L C}\right.$ and $O_{R}$ are the observability matrices of the pairs $\left(A-L C, e_{1}\right),\left(R, e_{2}\right)$ respectively) and $F=S^{-1} L$.

Another approach consists in a positive representation of (14) in original coordinates in the sense of Theorem 2.

Theorem 5. [38], [37], [39] Let Assumption 2 be satisfied, $x \in \mathcal{L}_{\infty}^{n}$ in (14) and there exist a matrix L $\in \mathbb{R}^{n \times p}$ such that the matrix

$$
\Sigma=\left[\begin{array}{ll}
D^{+} & D^{-} \\
D^{-} & D^{+}
\end{array}\right]
$$

is Schur stable for $D=A-L C$. Then for all $t \in \mathbb{Z}_{+}$the estimates $\underline{x}_{t}, \bar{x}_{t}$ are bounded and the relations (15) are satisfied for

$$
\begin{gathered}
\underline{x}_{t+1}=D^{+} \underline{x}_{t}-D^{-} \bar{x}_{t}+L y_{t}-|L| E_{p} V+\underline{d}_{t} \\
\bar{x}_{t+1}=D^{+} \bar{x}_{t}-D^{-} \underline{x}_{t}+L y_{t}+|L| E_{p} V+\bar{d}_{t} \\
\underline{x}_{0}=\underline{X}_{0}, \bar{x}_{0}=\bar{X}_{0} .
\end{gathered}
$$


Proof. The system (14) can be rewritten as follows:

$$
\begin{aligned}
x_{t+1} & =D x_{t}+L\left(y_{t}-v_{t}\right)+d_{t} \\
& =\left[D^{+}-D^{-}\right] x_{t}+L\left(y_{t}-v_{t}\right)+d_{t} .
\end{aligned}
$$

Then the dynamics of the errors $\underline{e}_{t}=x_{t}-\underline{x}_{t}, \bar{e}_{t}=\bar{x}_{t}-x_{t}$ obey the equations:

$$
\begin{aligned}
& \underline{e}_{t+1}=D^{+} \underline{e}_{t}+D^{-} \bar{e}_{t}+|L| E_{p} V-L v_{t}+d_{t}-\underline{d}_{t}, \\
& \bar{e}_{t+1}=D^{+} \bar{e}_{t}+D^{-} \underline{e}_{t}+|L| E_{p} V-L v_{t}+\bar{d}_{t}-d_{t} .
\end{aligned}
$$

By definition, the matrices $D^{+}, D^{-}$are nonnegative. According to Assumption 2 the inputs $|L| E_{p} V-L v_{t}+d_{t}-\underline{d}_{t},|L| E_{p} V-$ $L v_{t}+\bar{d}_{t}-d_{t}$ are also nonnegative, thus the estimation error dynamics is cooperative and starting from $\underline{e}_{0} \geq 0, \bar{e}_{0} \geq 0$ we get that $\underline{e}_{t} \geq 0, \bar{e}_{t} \geq 0$ for all $t \in \mathbb{Z}_{+}$. Since the matrix $\Sigma$ is Schur stable and the inputs are bounded, the variables $\underline{e}_{t}, \bar{e}_{t}$ possess the same property, that implies boundedness of $\underline{x}_{t}, \bar{x}_{t}$.

According to [40] a nonnegative matrix $\Sigma$ is Schur stable if there exists a diagonal positive definite matrix $P_{\Sigma} \in \mathbb{R}^{2 n \times 2 n}$ such that $\Sigma^{\mathrm{T}} P_{\Sigma} \Sigma-P_{\Sigma} \prec 0$, or this condition can be replaced with existence of a vector $p_{\Sigma} \in \mathbb{R}_{+}^{2 n}, p_{\Sigma}>0$ such that $p_{\Sigma}^{\mathrm{T}}\left(\Sigma-I_{2 n}\right)<0$. It is possible to show [37] that due to block symmetric structure of the matrix $\Sigma$ and its non-negativeness, the stability of $\Sigma$ is equivalent to stability of the matrix $|D|=D^{+}+D^{-}$.

Extension of these results for time-varying discrete-time systems is given in [41], where also positive representation is considered for periodical systems.

Remark 2. It is worth to stress that $d$ could be a function of the state $x$ provided that there exist known bounded signals $\bar{d}, \underline{d}$ satisfying Assumption 2. Therefore, the presented interval observers (16), (19) and (20) can be applied to nonlinear systems in the output canonical form, for instance. Application of these results to nonlinear systems is illustrated on examples below.

Example 2. Consider the following system [37]:

$$
\begin{aligned}
x_{t+1} & =A x_{t}+d_{t}, y_{t}=C x_{t}+v_{t}, \\
d_{t} & =b_{t}+\delta b\left(t, x_{t}\right),
\end{aligned}
$$

where $x_{t} \in \mathbb{R}^{2}, y_{t} \in \mathbb{R}, v_{t} \in \mathbb{R}$ are respectively the state, the output and the measurement noise $(\|v\| \leq V=0.1$, for simulation we used $v(t)=V \sin (t)$ ). The signals

$$
\begin{aligned}
b_{t} & =[\sin (0.1 t) \cos (0.2 t)]^{\mathrm{T}} \\
\delta b\left(t, x_{t}\right) & =\delta\left[\sin \left(0.5 t x_{2 t}\right) \sin (0.3 t)\right]^{\mathrm{T}}
\end{aligned}
$$

are the known portion $b$ and uncertain deviation $\delta b$ from the nominal $b$, for which we know that it is bounded by $\delta=0.5$. Thus $\underline{d}_{t}=b_{t}-\delta, \bar{d}(t)=b_{t}+\delta$ and Assumption 2, is satisfied. Finally,

$$
A=\left[\begin{array}{ll}
0.3 & -0.7 \\
0.6 & -0.5
\end{array}\right], C=\left[\begin{array}{ll}
1 & 0
\end{array}\right],
$$

and there is no observer gain $L \in \mathbb{R}^{2}$ making the matrix $D=A-L C$ nonnegative. The matrix $A$ is Schur stable (it has complex eigenvalues), but the matrix $|A|=A^{+}+A^{-}$is unstable. Applying Matlab YALMIP toolbox we obtain

$$
L=\left[\begin{array}{ll}
0.3 & 0.6
\end{array}\right]^{\mathrm{T}},
$$

then $\lambda(D)=\left[\begin{array}{ll}0 & -0.5\end{array}\right]^{\mathrm{T}}$ and $\lambda(|D|)=\left[\begin{array}{ll}0 & 0.5\end{array}\right]^{\mathrm{T}}$, therefore all conditions of Theorem 5 are satisfied for this choice of $L$. The matrices

$$
R=\left[\begin{array}{cc}
-0.5 & 0 \\
1 & 0
\end{array}\right], S=\left[\begin{array}{cc}
0.609 & 0.814 \\
-1.162 & 0.581
\end{array}\right]
$$

satisfy to all conditions of Theorem 4. Therefore now we can apply both observers (19) and (20) in this examples. The results 

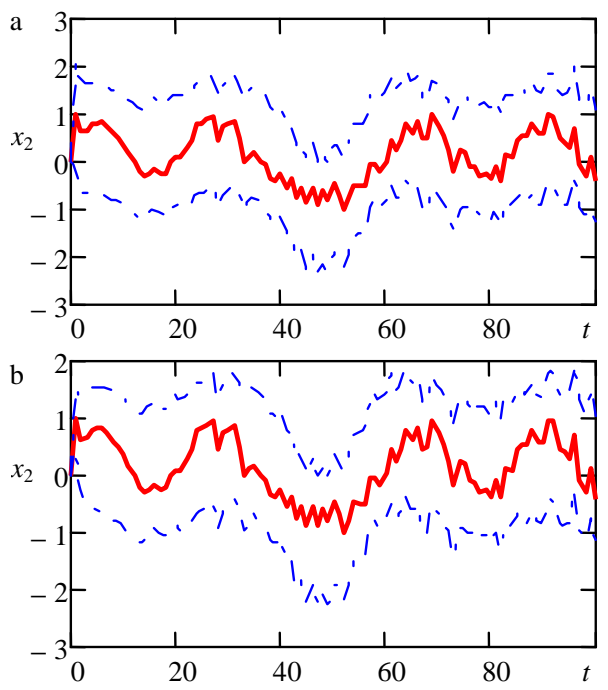

Figure 2. Comparison of interval estimation performed by the observer (19) (part (a)) and (20) (part (b)) [37]

of simulation for the interval estimation of the unmeasured coordinate $x_{2}$ are shown in Fig. 2. As we can conclude in this examples the observers demonstrate a similar performance.

Example 3. Consider a variant of the Hénon model [37]:

$$
\begin{aligned}
x_{t+1} & =A x_{t}+r\left[1-a(t) x_{1 t}^{2}+b_{t}\right], \\
y_{t} & =x_{1 t}+v_{t},
\end{aligned}
$$

where $x_{t} \in \mathbb{R}^{2}$ is the state, $y_{t} \in \mathbb{R}$ is the output, $v_{t} \in \mathbb{R}$ is the measurement noise $(\|v\| \leq V=0.1)$ and $b_{t} \in \mathbb{R}$ is the disturbance $(\|b\| \leq \delta=0.015)$,

$$
\begin{gathered}
A=\left[\begin{array}{cc}
0 & 1 \\
0.3 & 0
\end{array}\right], r=\left[\begin{array}{l}
1 \\
0
\end{array}\right], \underline{a} \leq a(t) \leq \bar{a}, \\
\underline{a}=1, \bar{a}=1.4 .
\end{gathered}
$$

The system has an uncertain parameter $a(t)$ with the values from an interval. If $a(t)=\bar{a}$, then the system equations become identical to the chaotic Hénon model. Thus assume that $\|x\|<+\infty$. Let us rewrite the system as follows:

$$
x_{t+1}=A x_{t}+d_{t}, d_{t}=r\left[1-a(t)\left[y_{t}-v_{t}\right]^{2}+b_{t}\right]
$$

then clearly it is in the form (14) and Assumption 2 is valid for

$$
\begin{aligned}
& \underline{d}_{t}=r\left[1-\bar{a} y_{t}^{2}-2 \bar{a}\left|y_{t}\right| V-\bar{a} V^{2}-\delta\right], \\
& \bar{d}_{t}=r\left[1-\underline{a} y_{t}^{2}+2 \bar{a}\left|y_{t}\right| V-\underline{a} V^{2}+\delta\right] .
\end{aligned}
$$

Finally, all conditions of Theorem 3 are satisfied for $L=[-0.10 .1]^{\mathrm{T}}$ and $C=\left[\begin{array}{ll}10 & 0\end{array}\right.$ (the matrix $A-L C$ is Schur stable and nonnegative) and the interval observer (16) solves the problem of interval state estimation. The results of simulation are shown in Fig 3.

\section{INTERVAL OBSERVERS FOR TIME-DELAY SYSTEMS}

Consider the system (6) equipped with an output $y \in \mathbb{R}^{p}$ available for measurements with a noise $v \in \mathcal{L}_{\infty}^{p}$ :

$$
y=C x, \quad \psi=y+v(t)
$$

where $C \in \mathbb{R}^{p \times n}$. Below the relation $a \leq b$ for $a, b \in \mathcal{C}_{\tau}^{n}$ is understood in the sense that $a(\theta) \leq b(\theta)$ for all $\theta \in[-\tau, 0]$. 

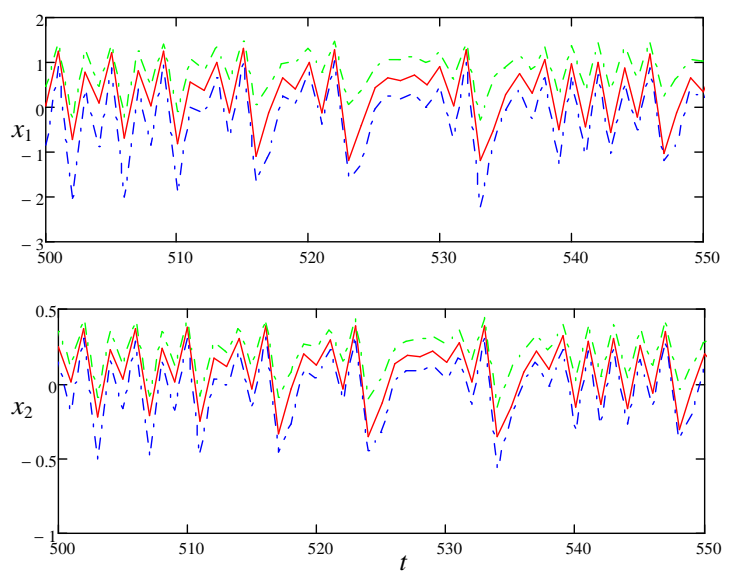

Figure 3. The results of simulation for Hénon model [37]

Assumption 3. Let $x \in \mathcal{L}_{\infty}^{n}$ with $\underline{x}_{0} \leq x_{t_{0}} \leq \bar{x}_{0}$ for some $\underline{x}_{0}, \bar{x}_{0} \in \mathcal{C}_{\tau}^{n}$; $\|v\| \leq V$ for a given $V>0$; and $\underline{\omega}(t) \leq \omega(t) \leq \bar{\omega}(t)$ for all $t \geq t_{0}$ for some known $\underline{\omega}, \bar{\omega} \in \mathcal{L}_{\infty}^{n}$.

In this assumption it is supposed that the state of the system (6) is bounded with an unknown upper bound, but with a specified admissible set for initial conditions $\left[\underline{x}_{0}, \bar{x}_{0}\right]$. Uncertainty of the system is collected in the external input $\omega$ with known bounds on the incertitude $\underline{\omega}, \bar{\omega}$, the measurement noise $v$ and the interval of initial conditions $\left[\underline{x}_{0}, \bar{x}_{0}\right]$.

As we see above, interval observers have an enlarged dimension (the examples given above have $2 n$ variables to estimate $n$ states). Thus, design of reduced order interval observers is of a big importance for applications. A reduced order interval observer for time-delay system (6), (21) has been proposed in [42], [43], those ideas are explained below.

For the system (6), (21) there exists a nonsingular matrix $S \in \mathbb{R}^{n \times n}$ such that $x=S\left[y^{\mathrm{T}} z^{\mathrm{T}}\right]^{\mathrm{T}}$ for an auxiliary variable $z \in \mathbb{R}^{n-p}$ (define $S^{-1}=\left[C^{\mathrm{T}} Z^{\mathrm{T}}\right]^{\mathrm{T}}$ for a matrix $Z \in \mathbb{R}^{(n-p) \times n}$ ), then

$$
\begin{aligned}
& \dot{y}(t)=R_{1} y(t)+R_{2} z(t)+\sum_{i=1}^{N}\left[D_{1 i} y\left(t-\tau_{i}\right)+D_{2 i} z\left(t-\tau_{i}\right)\right]+C \omega(t), \\
& \dot{z}(t)=R_{3} y(t)+R_{4} z(t)+\sum_{i=1}^{N}\left[D_{3 i} y\left(t-\tau_{i}\right)+D_{4 i} z\left(t-\tau_{i}\right)\right]+Z \omega(t),
\end{aligned}
$$

for some matrices $R_{k}, D_{k i}, k=\overline{1,4}, i=\overline{1, N}$ of appropriate dimensions. Introducing a new variable $w=z-K y=U x$ for a matrix $K \in \mathbb{R}^{(n-p) \times p}$ with $U=Z-K C$, from (22) the following equation is obtained

$$
\begin{aligned}
\dot{w}(t) & =G_{0} \psi(t)+M_{0} w(t)+\sum_{i=1}^{N}\left[G_{i} \psi\left(t-\tau_{i}\right)+M_{i} w\left(t-\tau_{i}\right)\right] \\
& +\beta(t), \beta(t)=U \omega(t)-G_{0} v(t)-\sum_{i=1}^{N} G_{i} v\left(t-\tau_{i}\right),
\end{aligned}
$$

where $\psi(t)$ is defined in (21), $G_{0}=R_{3}-K R_{1}+\left(R_{4}-K R_{2}\right) K, M_{0}=R_{4}-K R_{2}$, and $G_{i}=D_{3 i}-K D_{1 i}+\left\{D_{4 i}-K D_{2 i}\right\} K$, $M_{i}=D_{4 i}-K D_{2 i}$ for $i=\overline{1, N}$. Under Assumption 3 and using the relations (3) the following inequalities follow:

$$
\begin{gathered}
\underline{\beta}(t) \leq \beta(t) \leq \bar{\beta}(t), \\
\underline{\beta}(t)=U^{+} \underline{\omega}(t)-U^{-} \bar{\omega}(t)-\sum_{i=0}^{N}\left|G_{i}\right| E_{p} V, \\
\bar{\beta}(t)=U^{+} \bar{\omega}(t)-U^{-} \underline{\omega}(t)+\sum_{i=0}^{N}\left|G_{i}\right| E_{p} V .
\end{gathered}
$$


Then the next interval reduced-order observer can be proposed for (6):

$$
\begin{gathered}
\underline{\dot{w}}(t)=G_{0} \psi(t)+M_{0} \underline{w}(t)+\sum_{i=1}^{N}\left[G_{i} \psi\left(t-\tau_{i}\right)+M_{i} \underline{w}\left(t-\tau_{i}\right)\right]+\underline{\beta}(t), \\
\dot{\bar{w}}(t)=G_{0} \psi(t)+M_{0} \bar{w}(t)+\sum_{i=1}^{N}\left[G_{i} \psi\left(t-\tau_{i}\right)+M_{i} \bar{w}\left(t-\tau_{i}\right)\right]+\bar{\beta}(t), \\
\underline{w}_{0}=U^{+} \underline{x}_{0}-U^{-} \bar{x}_{0}, \bar{w}_{0}=U^{+} \bar{x}_{0}-U^{-} \underline{x}_{0} .
\end{gathered}
$$

The applicability conditions for (24) are given below.

Theorem 6. [42] Let Assumption 3 be satisfied and the matrices $M_{0}, M_{i}, i=\overline{1, N}$ form an asymptotically stable cooperative system (see lemmas 5 and 6). Then $\underline{x}, \bar{x} \in \mathcal{L}_{\infty}^{n}$ and

$$
\underline{x}(t) \leq x(t) \leq \bar{x}(t) \quad \forall t \geq 0
$$

where

$$
\begin{gathered}
\underline{x}(t)=S^{+}\left[\underline{y}(t)^{T} \underline{z}(t)^{T}\right]^{T}-S^{-}\left[\bar{y}(t)^{T} \bar{z}(t)^{T}\right]^{T}, \\
\bar{x}(t)=S^{+}\left[\bar{y}(t)^{T} \bar{z}(t)^{T}\right]^{T}-S^{-}\left[\underline{y}(t)^{T} \underline{z}(t)^{T}\right]^{T}, \\
\underline{y}(t)=\psi(t)-V, \bar{y}(t)=\psi(t)+V, \\
\underline{z}(t)=\underline{w}(t)+K^{+} \underline{y}-K^{-} \bar{y}, \bar{z}(t)=\bar{w}(t)+K^{+} \bar{y}-K^{-} \underline{y} .
\end{gathered}
$$

Proof. From the theorem conditions the matrix $M_{0}$ is Metzler and the matrices $M_{i}, i=\overline{1, N}$ are nonnegative, in addition from Lemma 6 there exist some $p, q \in \mathbb{R}_{+}^{n-p}(p>0$ and $q>0)$ such that

$$
p^{\mathrm{T}} \sum_{i=0}^{N} M_{i}=-q^{\mathrm{T}}
$$

Consider two estimation errors $\underline{e}=w-\underline{w}, \bar{e}=\bar{w}-w$ :

$$
\begin{aligned}
& \underline{\dot{e}}(t)=M_{0} \underline{e}(t)+\sum_{i=1}^{N} M_{i} \underline{e}\left(t-\tau_{i}\right)+\underline{d}(t), \\
& \dot{\bar{e}}(t)=M_{0} \bar{e}(t)+\sum_{i=1}^{N} M_{i} \bar{e}\left(t-\tau_{i}\right)+\bar{d}(t),
\end{aligned}
$$

where $\underline{d}(t)=\beta(t)-\underline{\beta}(t)$ and $\bar{d}(t)=\bar{\beta}(t)-\beta(t)$. By definition of $\underline{\beta}, \bar{\beta}$ the signals $\underline{d}, \bar{d} \in \mathbb{R}_{+}^{n-p}$, therefore $\underline{e}(t), \bar{e}(t) \in \mathcal{C}_{\tau+}^{n-p}$ for all $t>0$ provided that $\underline{e}(0), \bar{e}(0) \in \mathcal{C}_{\tau+}^{n-p}$, the last relation is satisfied by the definition of $\underline{w}_{0}$ and $\bar{w}_{0}$. Note that the expressions for $\underline{x}(t), \bar{x}(t)$ follow the relations (3). To prove that the errors $\underline{e}, \bar{e}$ are bounded, as in [30], consider for (27) the Lyapunov functional $V: \mathcal{C}_{\tau+}^{n} \rightarrow \mathbb{R}_{+}$defined as

$$
V(\varphi)=p^{T} \varphi(0)+\sum_{i=1}^{N} \int_{-\tau_{i}}^{0} p^{T} M_{i} \varphi(s) d s .
$$

Let us stress that for any $\varphi \in \mathcal{C}_{\tau+}^{n}$ the functional $V$ is positive definite and radially unbounded, its derivative for $\underline{e}$ takes the form (for $\bar{e}$ the analysis is the same):

$$
\begin{aligned}
\dot{V} & =p^{T}\left[M_{0} \underline{e}(t)+\sum_{i=1}^{N} M_{i} \underline{e}\left(t-\tau_{i}\right)+\underline{d}(t)\right]+\sum_{i=1}^{N} p^{T} M_{i}\left[\underline{e}(t)-\underline{e}\left(t-\tau_{i}\right)\right] \\
& =p^{T}\left[\sum_{i=0}^{N} M_{i} \underline{e}(t)+\underline{d}(t)\right] \leq-q^{T} \underline{e}(t)+p^{T} \underline{d}(t) .
\end{aligned}
$$

Thus for $\underline{d}=0$ the system is globally asymptotically stable, and since $\underline{d} \in \mathcal{L}_{\infty}^{n-p}$ (by construction and Assumption 3 ) one finds 
that the error $\underline{e}$ is bounded (see [44] or [45] for the proof that in fact the system is input-to-state stable).

The main condition of Theorem 6 is rather straightforward: the matrices $M_{0}, M_{i}, i=\overline{1, N}$ have to form a stable cooperative system. It is a standard LMI problem to find a matrix $K$ such that the system composed by $M_{0}, M_{i}, i=\overline{1, N}$ is stable, but to find a matrix $K$ making the system stable and cooperative simultaneously could be more complicated. However, the advantage of Theorem 6 is that its main condition can be reformulated using LMIs following the idea of [46].

Proposition 1. [42] Let there exist $\varsigma \in \mathbb{R}_{+}, p \in \mathbb{R}_{+}^{n-p}, q \in \mathbb{R}_{+}^{n-p}$ and $B \in \mathbb{R}^{(n-p) \times p}$ such that the following LMIs are satisfied:

$$
\begin{gathered}
p^{\mathrm{T}} \Pi_{0}-E_{n-p}^{\mathrm{T}} B \Pi_{1}+q^{\mathrm{T}} \leq 0, p>0, q>0 ; \quad \operatorname{diag}[p] R_{4}-B R_{2}+\varsigma I_{n-p} \geq 0, \varsigma>0 \\
\operatorname{diag}[p] D_{4 i}-B D_{2 i} \geq 0, i=\overline{1, N} ; \quad \Pi_{0}=R_{4}+\sum_{i=1}^{N} D_{4 i}, \Pi_{1}=R_{2}+\sum_{i=1}^{N} D_{2 i}
\end{gathered}
$$

then $K=\operatorname{diag}[p]^{-1} B$ and the matrices $M_{0}=R_{4}-K R_{2}, M_{i}=D_{4 i}-K D_{2 i}, i=\overline{1, N}$ represent a stable cooperative system in (24).

If these LMIs are not satisfied, the assumption that the matrix $M_{0}$ is Metzler and the matrices $M_{i}, i=\overline{1, N}$ are nonnegative can be relaxed using Lemma 8. According to Lemma 8 there exists a coordinate transformation $\varrho=P w$ that maps $M_{0}$ to a Metzler matrix $P M_{0} P^{-1}$, but Lemma 6 also requires the transformed matrices $P M_{i} P^{-1}$ to be nonnegative, that is hard to satisfy. Fortunately, using the ideas of theorems 2 and 5 the non-negativity of $P M_{i} P^{-1}$ is not necessary, and its nonnegative representation can be used [42].

This approach can also be applied to the system (6) with time-varying and uncertain delays $\tau_{i}: \mathbb{R} \rightarrow[-\tau, 0]$ :

$$
\begin{gathered}
\dot{x}(t)=A_{0} x(t)+\sum_{i=1}^{N} A_{i} x\left(t-\tau_{i}(t)\right)+b(t), \\
\underline{\tau}_{i} \leq \tau_{i}(t) \leq \bar{\tau}_{i} \quad t \geq 0, i=\overline{1, N}
\end{gathered}
$$

with $\tau=\max _{1 \leq i \leq N} \bar{\tau}_{i}$ for some given $\underline{\tau}_{i}, \bar{\tau}_{i} \in \mathbb{R}_{+}$, then applying the same transformations of coordinates to (28) we obtain:

$$
\dot{\varrho}(t)=T_{0} \psi(t)+Y_{0} \varrho(t)+\sum_{i=1}^{N}\left[T_{i} \psi\left\{t-\tau_{i}(t)\right\}+Y_{i} \varrho\left\{t-\tau_{i}(t)\right\}\right]+\gamma(t),
$$

where $Y_{i}=P M_{i} P^{-1}, T_{i}=P G_{i}$ for $i=\overline{0, N}, \gamma(t)=P \beta(t)$ and

$$
\underline{\gamma}(t)=P^{+} \underline{\beta}(t)-P^{-\bar{\beta}}(t), \bar{\gamma}(t)=P^{+} \bar{\beta}(t)-P^{-} \underline{\beta}(t) .
$$

Next, the idea is to replace the delayed term $\varrho\left\{t-\tau_{i}\right\}$ with its minimum and maximum over the interval $\left[\underline{\tau}_{i}, \bar{\tau}_{i}\right]$ :

$$
\left.\left.\underline{m}_{i}[\varrho(t)]\right)=\min _{s \in\left[\underline{\tau}_{i}, \bar{\tau}_{i}\right]} \varrho(t-s), \bar{m}_{i}[\varrho(t)]\right)=\max _{s \in\left[\underline{\tau}_{i}, \bar{\tau}_{i}\right]} \varrho(t-s),
$$

that does not influence on the possibility of interval estimation. Thus the observer equations can be rewritten as follows:

$$
\begin{aligned}
\underline{\dot{\varrho}}(t) & =T_{0} \psi(t)+Y_{0} \underline{\varrho}(t)+\sum_{i=1}^{N}\left\{T_{i}^{+} \underline{m}_{i}[\psi(t)]-T_{i}^{-} \bar{m}_{i}[\psi(t)]\right. \\
& \left.+Y_{i}^{+} \underline{m}_{i}[\underline{\varrho}(t)]-Y_{i}^{-} \bar{m}_{i}[\bar{\varrho}(t)]\right\}+\underline{\gamma}(t), \\
\dot{\bar{\varrho}}(t) & =T_{0} \psi(t)+Y_{0} \bar{\varrho}(t)+\sum_{i=1}^{N}\left\{T_{i}^{+} \bar{m}_{i}[\psi(t)]-T_{i}^{-} \underline{m}_{i}[\psi(t)]\right. \\
& \left.+Y_{i}^{+} \bar{m}_{i}[\bar{\varrho}(t)]-Y_{i}^{-} \underline{m}_{i}[\underline{\varrho}(t)]\right\}+\bar{\gamma}(t), \\
& \underline{\varrho}_{0}=O^{+} \underline{x}_{0}-O^{-} \bar{x}_{0}, \bar{\varrho}_{0}=O^{+} \bar{x}_{0}-O^{-} \underline{x}_{0},
\end{aligned}
$$

where $O=P U$. It is worth to stress that the observer (29) is nonlinear. 
Theorem 7. [42] Let assumption 3 be satisfied for (28). Then

$$
\underline{x}(t) \leq x(t) \leq \bar{x}(t)
$$

for all $t \geq 0$, where $\underline{x}(t), \bar{x}(t)$ are defined by (25), (26) and

$$
\underline{w}(t)=\left[P^{-1}\right]^{+} \underline{\varrho}-\left[P^{-1}\right]^{-} \bar{\varrho}, \bar{w}(t)=\left[P^{-1}\right]^{+} \bar{\varrho}-\left[P^{-1}\right]^{-} \underline{\varrho} .
$$

The cases of delayed measurements are studied in [47], [43]. An extension to descriptor delay systems is presented in [48].

Example 4. Following [49], [50], consider a nonlinear model of testosterone dynamics with an external impulsive input:

$$
\begin{aligned}
\dot{R}(t) & =\frac{A}{K+T\left(t-\tau_{0}(t)\right)^{\mu}}-b_{1} R(t)+d(t), \\
\dot{L}(t) & =g_{1} R\left(t-\tau_{1}\right)-b_{2} L(t) \\
\dot{T}(t) & =g_{2} L\left(t-\tau_{2}\right)-b_{3} T(t)
\end{aligned}
$$

where $R \in \mathbb{R}_{+}$is the concentration of hypothalamic hormone $(\mathrm{GnRH}), L \in \mathbb{R}_{+}$is the concentration of pituitary hormone (LH) and $T \in \mathbb{R}_{+}$is the testosterone concentration (Te), $b_{1}=b_{2}=b_{3}=1, g_{1}=10, g_{2}=50, \tau_{1}=1, \tau_{2}=2$ and the system (30) uncertainty is represented by the nonlinear function parameters

$$
\begin{gathered}
8=\underline{A} \leq A \leq \bar{A}=12,1.5=\underline{\mu} \leq \mu \leq \bar{\mu}=2.5, \\
1.5=\underline{K} \leq K \leq \bar{K}=2.5,1=\underline{\tau}_{0} \leq \tau_{0} \leq \bar{\tau}_{0}=2 .
\end{gathered}
$$

For numerical simulation the values $A=10, \mu=2, K=2$ and $\tau_{0}(t)=1.5+0.5 \sin (0.1 t)$ have been used. The input $d(t) \in \mathbb{R}_{+}$represents a pulsatile feedback mechanism from the testosterone serum to the hypothalamic hormone [49], this input is a multiplication of two signals

$$
d(t)=d_{0}(t) \delta d(t)
$$

where $d_{0}$ is the known part of the feedback generating the pulses $\left(d_{0}(t)=(1+\sin (0.1 t)) e^{-\bmod [t, 5+5 \sin (0.01 t)]^{2}}\right.$ for simulation) and $\delta d$ is unknown modulating signal (for numerical experiments $\delta d(t)=1-\delta \cos (2 t), \delta=0.25$ ). For these parameters the model (30) has bounded solutions and Assumption 3 is satisfied. It is assumed that the testosterone concentration $T(t)$ is available from the direct measurements.

Denote $w=[R L]^{\mathrm{T}}$ then [42]:

$$
\dot{w}(t)=M_{0} w(t)+M_{1} w\left(t-\tau_{1}\right)+\beta(t),
$$

where $\beta(t)=\left[\frac{A}{K+T\left(t-\tau_{0}(t)\right)^{\mu}}+d(t) 0\right]^{\mathrm{T}}$ and

$$
M_{0}=\left[\begin{array}{cc}
-b_{1} & 0 \\
0 & -b_{2}
\end{array}\right], M_{1}=\left[\begin{array}{cc}
0 & 0 \\
g_{1} & 0
\end{array}\right]
$$

The direct computations give

$$
\begin{aligned}
& \underline{\beta}(t)=\left[\begin{array}{c}
\frac{A}{\bar{K}+\max \left\{\underline{\phi}\left(\underline{m}_{0}[T(t)]\right), \underline{\phi}\left(\bar{m}_{0}[T(t)]\right)\right\}}+d_{0}(t)(1-\delta) \\
0
\end{array}\right], \bar{\beta}(t)=\left[\begin{array}{c}
\frac{\bar{A}}{\underline{K}+\min \left\{\bar{\phi}\left(\underline{m}_{0}[T(t)]\right), \bar{\phi}\left(\bar{m}_{0}[T(t)]\right)\right\}}+d_{0}(t)(1+\delta) \\
0
\end{array}\right], \\
& \underline{\phi}(y)=\left\{\begin{array}{ll}
y^{\bar{\mu}} & \text { if } y>1 ; \\
y^{\underline{\mu}} & \text { if } y \leq 1,
\end{array} \quad \bar{\phi}(y)= \begin{cases}y^{\underline{\mu}} & \text { if } y>1 \\
y^{\bar{\mu}} & \text { if } y \leq 1\end{cases} \right.
\end{aligned}
$$

where $\underline{m}_{0}[T(t)]=\min _{s \in\left[\underline{\tau}_{0}, \bar{\tau}_{0}\right]} T(t-s), \bar{m}_{0}[T(t)]=\max _{s \in\left[\underline{\tau}_{0}, \bar{\tau}_{0}\right]} T(t-s)$ as before. The results of the interval reduced-order observer simulation are presented in Fig. 4 (the solid lines represent the concentrations $R$ and $L$, the dash lines are used for the interval estimates). 

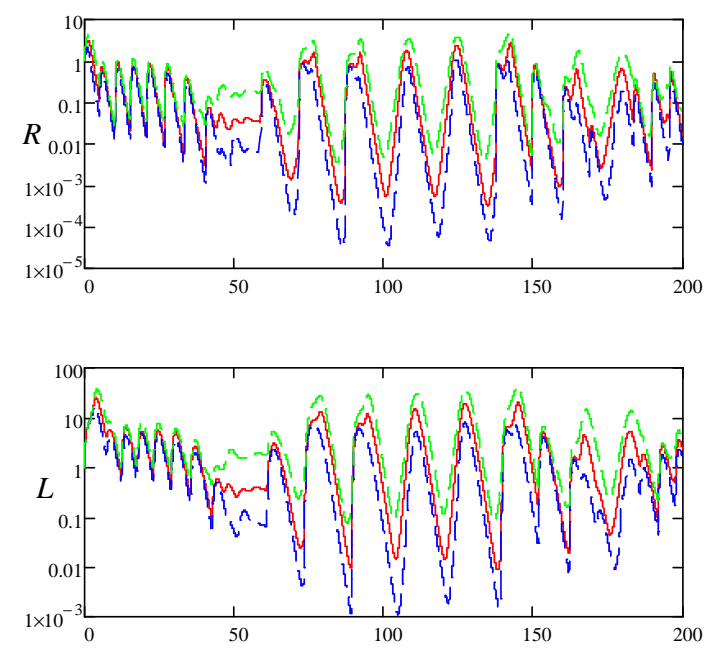

Figure 4. Interval estimation for the testosterone model (30) [42]

\section{THE CASE OF LPV MODELS}

Consider a LPV system:

$$
\dot{x}=\left[A_{0}+\Delta A(\rho(t))\right] x+d(t), y=C x+v(t), t \geq 0,
$$

where $x \in \mathbb{R}^{n}$ is the state, $y \in \mathbb{R}^{p}$ is the output available for measurements, $\rho(t) \in \Pi \subset \mathbb{R}^{r}$ is the vector of scheduling parameters, $\rho \in \mathcal{L}_{\infty}^{r}$. The values of the scheduling vector $\rho$ are not available for measurements, and only the set of admissible values $\Pi$ is known. The matrices $A_{0} \in \mathbb{R}^{n \times n}$ and $C \in \mathbb{R}^{p \times n}$ are known, the matrix function $\Delta A: \Pi \rightarrow \mathbb{R}^{n \times n}$ is piecewise continuous and also known for a given value of $\rho$. The signals $d: \mathbb{R}_{+} \rightarrow \mathbb{R}^{n}$ and $v: \mathbb{R}_{+} \rightarrow \mathbb{R}^{p}$ are the external input and measurement noise respectively.

Assumption 4. $x \in \mathcal{L}_{\infty}^{n}$ and $x(0) \in\left[\underline{x}_{0}, \bar{x}_{0}\right]$ for some known $\underline{x}_{0}, \bar{x}_{0} \in \mathbb{R}^{n} ; \underline{d}(t) \leq d(t) \leq \bar{d}(t)$ and $|v(t)| \leq V$ for all $t \geq 0$ and some known $\underline{d}, \bar{d} \in \mathcal{L}_{\infty}^{n}$ and $V>0 ; \underline{\Delta A} \leq \Delta A(\rho) \leq \overline{\Delta A}$ for all $\rho \in \Pi$ and some known $\underline{\Delta A}, \overline{\Delta A} \in \mathbb{R}^{n \times n}$.

In this case the system (31) uncertainty is presented by the interval of initial conditions $\left[\underline{x}_{0}, \bar{x}_{0}\right]$, the measurement noise $v(t)$ with an upper bound $V$, the input $d(t)$ from a known bounded interval $[\underline{d}(t), \bar{d}(t)]$ for all $t \in \mathbb{R}_{+}$, the matrix $\Delta A(\rho)$ from the interval $[\underline{\Delta A}, \overline{\Delta A}]$ for all $t \geq 0$. The interval $[\underline{\Delta A}, \overline{\Delta A}]$ is easy to compute for a given set $\Pi$ and known $\Delta A(\rho)$ (in a polytopic case, for example).

The objective is to design an interval observer for the system (31). Before introduction of interval observer equations note that for a matrix $L \in \mathbb{R}^{n \times p}$ the system (31) can be rewritten as follows:

$$
\dot{x}=\left[A_{0}-L C\right] x+\Delta A(\rho(t)) x+L[y-v(t)]+d(t),
$$

and according to Lemma 1 and Assumption 4 we have for all $\rho \in \Pi$ :

$$
\begin{gathered}
\underline{\Delta A^{+}} \underline{x}^{+}-\overline{\Delta A} \underline{x}^{-}-\underline{\Delta A^{-}} \bar{x}^{+}+\overline{\Delta A} \bar{x}^{-} \leq \Delta A(\rho) x \\
\leq \overline{\Delta A} \bar{x}^{+}-\underline{\Delta A^{+} \bar{x}^{-}-\overline{\Delta A} \underline{x}^{+}+\underline{\Delta A^{-}} \underline{x}^{-}}
\end{gathered}
$$

provided that $\underline{x} \leq x \leq \bar{x}$ for some $\underline{x}, \bar{x} \in \mathbb{R}^{n}$.

\section{A. Nonnegative $L P V$ systems}

In this case we will assume the following.

Assumption 5. $x(t) \in \mathbb{R}_{+}^{n}$ and $\underline{d}(t) \in \mathbb{R}_{+}^{n}$ for all $t \geq 0 ; \underline{\Delta A}=0$. 
Under assumptions 4 and 5 we also have that $d(t), \bar{d}(t) \in \mathbb{R}_{+}^{n}$ for all $t \geq 0$. Note that the condition $d(t) \in \mathbb{R}_{+}^{n}$ is required for the system (31) with $\Delta A(\rho(t)) \equiv 0$ to be nonnegative [24]. The last condition $\underline{\Delta A}=0$ simply means that $A_{0}$ is the minimal value of $A_{0}+\Delta A(\rho)$ for $\rho \in \Pi$ and $\overline{\Delta A} \geq 0$, this condition can be always satisfied after a suitable shift of $A_{0}, \underline{\Delta A}$ and $\overline{\Delta A}$.

Denote as usual by $\underline{x}(t)$ and $\bar{x}(t)$ the lower and upper bound estimates of the state $x(t)$ respectively. Let us introduce two observer gain matrices $\underline{L}, \bar{L} \in \mathbb{R}^{n \times p}$, whose values will be specified later, then a candidate interval observer for the nonnegative system (31) is given by [34]:

$$
\begin{aligned}
\underline{\dot{x}}= & {\left[A_{0}-\underline{L} C\right] \underline{x}+\max \left\{0, \underline{L} y-|\underline{L}| V E_{p}\right\}+\underline{d}(t), } \\
\dot{\bar{x}}= & {\left[A_{0}-\bar{L} C+\overline{\Delta A}\right] \bar{x}+\bar{L} y+|\bar{L}| V E_{p}+\bar{d}(t), } \\
& \underline{x}(0)=\underline{x}_{0}, \bar{x}(0)=\bar{x}_{0} .
\end{aligned}
$$

Note that it is a LTI system and a similar structure of interval observer has been proposed in [18], [51], where there is no $\max \{\cdot\}$ function in the first equation, whose introduction improves the estimation accuracy. Indeed, under conditions $\underline{L} C \geq 0$ and $x(t) \geq 0$, the item $\underline{L}[y(t)-v(t)]=\underline{L C x}(t)$ is nonnegative, but its accessible lower bound $\underline{L} y(t)-|\underline{L}| V E_{p}$ can take negative values in general, then introduction of the function $\max \{\cdot\}$ improves the tightness of the bound.

Theorem 8. [34] Let assumptions 4-5 be satisfied, the matrices $A_{0}-\underline{L} C, A_{0}-\bar{L} C$ be Metzler and the following constraints be verified for some $\lambda_{1}, \lambda_{2} \in \mathbb{R}_{+}^{n} \backslash\{0\}$ :

$$
\begin{gathered}
{\left[A_{0}-\underline{L} C\right]^{T} \lambda_{1}+Z^{T} E_{s}<0, \quad\left[A_{0}-\bar{L} C+\overline{\Delta A}\right]^{T} \lambda_{2}+Z^{T} E_{s}<0} \\
\lambda_{i}-\gamma E_{n}<0, i=1,2 ; \quad \underline{L} C \geq 0, \bar{L} C \geq 0
\end{gathered}
$$

for a scalar $\gamma>0$ and $Z \in \mathbb{R}_{+}^{s \times n}, 0<s \leq n$. Then the solutions of (31), (33) satisfy

$$
0 \leq \underline{x}(t) \leq x(t) \leq \bar{x}(t) \quad \forall t \geq 0
$$

and $\underline{x}, \bar{x} \in \mathcal{L}_{\infty}^{n}$. In addition, $L_{1}$ gain of the operators $\underline{d} \rightarrow Z \underline{x}$ and $\bar{d} \rightarrow Z \bar{x}$ is less than $\gamma$.

The matrix $Z$ and the $L_{1}$ gain stability conditions are introduced in order to be able to improve/regulate the accuracy of interval estimation for some part of variables (for example, the matrix $Z$ can select all state coordinates excluding the measured variables).

Note that the conditions of Theorem 8 imposed on the gains $\underline{L}, \bar{L}$ can be formulated in terms of the following linear programming problem for the case $C \geq 0$ (see also [51]): it is required to find $\lambda_{1}, \lambda_{2} \in \mathbb{R}^{n}, w_{1}, w_{2} \in \mathbb{R}_{+}^{p}$ and a diagonal matrix $S \in \mathbb{R}_{+}^{n \times n}$ such that [34]:

$$
\begin{gathered}
A_{0}^{\mathrm{T}} \lambda_{1}-C^{\mathrm{T}} w_{1}+Z^{\mathrm{T}} E_{s}<0, \quad\left[A_{0}+\overline{\Delta A}\right]^{\mathrm{T}} \lambda_{2}-C^{\mathrm{T}} w_{2}+Z^{\mathrm{T}} E_{s}<0, \\
\left|\lambda_{i}\right|=\gamma, \lambda_{i}>0, w_{i} \geq 0, i=1,2 ; \quad \underline{L}=\lambda_{1} w_{1}^{\mathrm{T}} \gamma^{-2}, \bar{L}=\lambda_{2} w_{2}^{\mathrm{T}} \gamma^{-2}, \\
A_{0}-\underline{L} C+S \geq 0, A_{0}-\bar{L} C+S \geq 0, S \geq 0 .
\end{gathered}
$$

Example 5. Following [52], [53] consider a model of droplet-based microfluidic system derived from the Chemical Master equation:

$$
\begin{aligned}
\dot{P}_{0} & =-\kappa(t) P_{0}, P_{0}(0)=1, \\
\dot{P}_{i} & =\kappa(t)\left[P_{i-1}-P_{i}\right], P_{i}(0)=0, i=\overline{1, N},
\end{aligned}
$$

where $P_{i}, i=\overline{0, N}$ is the probability that a droplet contains $i$ crystals, and $\kappa(t) d t$ is the probability that a critical nucleus will form during an infinitesimal time interval $d t$. This model evaluates the crystal growth process in time. According to [52] $\kappa(t)=J(S(t)) V(t)$, where $S(t)$ is the supersaturation and $V(t)$ is the droplet volume, both of them are assumed to be available from (noisy) measurements, but the function $J$ is not exactly known. Therefore, we will assume that for the function $\kappa(t)$ only a lower $\underline{\kappa}(t)$ and an upper $\bar{\kappa}(t)$ bounds are available. In addition, for simplicity of presentation we assume that the 


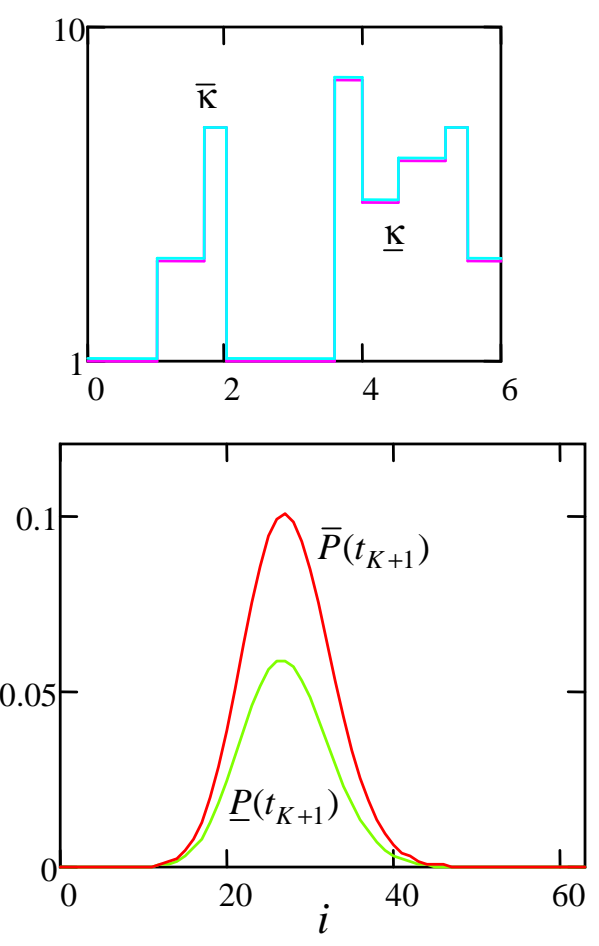

Figure 5. The results of simulations for a nonnegative microfluidic system [34]

functions $\underline{\kappa}(t), \bar{\kappa}(t)$ are piecewise constant, i.e. there exist intervals $\left[t_{j}, t_{j+1}\right), j=0, K$ such that $\underline{\kappa}(t)=\underline{\kappa}_{j}, \bar{\kappa}(t)=\bar{\kappa}_{j}$ for all $t \in\left[t_{j}, t_{j+1}\right), t_{0}=0$. For this system there is no measurement of the state $(C=0)$. It is a time-varying autonomous linear system, but since the exact value of $\kappa(t)$ is not known, then the LPV framework has to be used. One of the main difficulties with this system is that the number of subsystems $N$ (the possible of number of crystals in a droplet) can be sufficiently large. And the only way to predict/evaluate a possible state of the crystal growth process in a droplet is based on estimation for Chemical Master equation.

The observer (33) on each interval $\left[t_{j}, t_{j+1}\right), j=\overline{0, K}$ can be rewritten as follows [34]:

$$
\begin{aligned}
\underline{\dot{x}} & =A_{0} \underline{x}, \\
\dot{\bar{x}} & =\left[A_{0}+\overline{\Delta A}\right] \bar{x},
\end{aligned}
$$

where

$$
A_{0}=\left[\begin{array}{ccccc}
-\bar{\kappa}_{j} & 0 & \ldots & 0 & 0 \\
\underline{\kappa}_{j} & -\bar{\kappa}_{j} & \ldots & 0 & 0 \\
\vdots & \vdots & \ddots & \vdots & \vdots \\
0 & 0 & \ldots & \underline{\kappa}_{j} & -\bar{\kappa}_{j}
\end{array}\right], A_{0}+\overline{\Delta A}=\left[\begin{array}{ccccc}
-\underline{\kappa}_{j} & 0 & \ldots & 0 & 0 \\
\bar{\kappa}_{j} & -\underline{\kappa}_{j} & \ldots & 0 & 0 \\
\vdots & \vdots & \ddots & \vdots & \vdots \\
0 & 0 & \ldots & \bar{\kappa}_{j} & -\underline{\kappa}_{j}
\end{array}\right]
$$

and all conditions of Theorem 8 are satisfied for $\underline{L}=\bar{L}=0$ (no measurements). Thus, we can iteratively apply the obtained interval observer (33) on each interval $\left[t_{j}, t_{j+1}\right), j=0, K$ in order to reconstruct the distribution $P_{i}\left(t_{K+1}\right), i=\overline{0, N}$ at the end of the process of crystallization, starting from a fixed initial distribution $\left(P_{0}(0)=1\right.$ and $P_{i}(0)=0$ for $\left.i=\overline{1, N}\right)$.

For $\underline{\kappa}(t), \bar{\kappa}(t)$ given in the top of Fig. 5 and $N=64$ the results of interval estimation of $P_{i}\left(t_{K+1}\right), i=\overline{0, N}$ are shown in Fig 5. As we can conclude from these results, even a small difference in $\underline{\kappa}(t), \bar{\kappa}(t)$ may lead on a short time interval $\left(t_{K+1}=6\right)$ to a big deviations of $\underline{P}_{i}\left(t_{K+1}\right)$ and $\bar{P}_{i}\left(t_{K+1}\right), i=\overline{0, N}$.

\section{B. Transformation of coordinates}

The requirement that the matrices $A_{0}-\underline{L} C, A_{0}-\bar{L} C$ have to be Metzler can be relaxed by means of a change of coordinates $z=T x$ with a nonsingular matrix $T$ such that the matrices $\underline{F}=T\left(A_{0}-\underline{L} C\right) T^{-1}, \bar{F}=T\left(A_{0}-\bar{L} C\right) T^{-1}$ are Metzler using 
the results of lemmas 8 or 9 , or the following one.

Lemma 10. [54] Let $D \in \Xi \subset \mathbb{R}^{n \times n}$ be a matrix variable satisfying the interval constraints $\Xi=\left\{D \in \mathbb{R}^{n \times n}: D_{a}-\Delta \leq\right.$ $\left.D \leq D_{a}+\Delta\right\}$ for some $D_{a}^{T}=D_{a} \in \mathbb{R}^{n \times n}$ and $\Delta \in \mathbb{R}_{+}^{n \times n}$. If for some constant $\mu \in \mathbb{R}_{+}$and a diagonal matrix $\Upsilon \in \mathbb{R}^{n \times n}$ the Metzler matrix $Y=\mu E_{n \times n}-\Upsilon$ has the same eigenvalues as the matrix $D_{a}$, then there is an orthogonal matrix $S \in \mathbb{R}^{n \times n}$ such that the matrices $S^{T} D S$ are Metzler for all $D \in \Xi$ provided that $\mu>n\|\Delta\|_{\max }$.

This result was used in [54] to design interval observers for linear time-varying systems (for the case of a measured vector of scheduling parameters $\rho$ ).

Therefore, the matrix $T$ can be found using the results of Lemma 8 (looking for $\underline{L}=\bar{L}=L$ ) or Lemma 10 . Note that if we would like to preserve non-negativity of the new state vector $z$, then it is required to find a nonnegative matrix $T$. In this case for a matrix $L \in \mathbb{R}^{n \times p}$ and $T \geq 0$ the system (31) in the coordinates $z$ can be rewritten as follows:

$$
\dot{z}=T\left[A_{0}-L C\right] T^{-1} z+\Delta F(\rho(t)) x+T L[y-v(t)]+\beta(t),
$$

where $\beta(t)=T d(t)$ and $\Delta F(\rho(t))=T \Delta A(\rho(t))$ with $\underline{\beta}(t)=T \underline{d}(t), \bar{\beta}(t)=T \bar{d}(t)$ by Lemma $1,0 \leq \Delta F(\rho) \leq \overline{\Delta F}=T \overline{\Delta A}$ for all $\rho \in \Pi$. The interval observer (33) saves its structure in the new coordinates:

$$
\begin{aligned}
& \underline{\dot{z}}=\underline{F z}+T \max \left\{0, \underline{L} y-|\underline{L}| V E_{p}\right\}+\underline{\beta}(t), \\
& \dot{\bar{z}}=[\bar{F}+\overline{\Delta F}] \bar{z}+T\left(\bar{L} y+|\bar{L}| V E_{p}\right)+\bar{\beta}(t) .
\end{aligned}
$$

The stability conditions and the proof for this interval observer follows Theorem 8 . Unfortunately it may be hard to find a nonnegative matrix $T$ [55], in such a case the variable $z$ is not nonnegative and the results presented in the next subsection can be applied.

\section{Generic LPV systems}

For the case of a non positive LPV system (31), the following interval observer structure is proposed [34]:

$$
\begin{aligned}
\underline{\dot{x}}= & {\left[A_{0}-\underline{L} C\right] \underline{x}+\left[\underline{\Delta A}^{+} \underline{x}^{+}-\overline{\Delta A} \underline{x}^{-}\right.} \\
& \left.-\Delta A^{-} \bar{x}^{+}+\overline{\Delta A}^{-} \bar{x}^{-}\right]+\underline{L} y-|\underline{L}| V E_{p}+\underline{d}(t), \\
\dot{\bar{x}}= & {\left[A_{0}-\bar{L} C\right] \bar{x}+\left[\overline{\Delta A} \bar{x}^{+}-\underline{\Delta A^{+}} \bar{x}^{-}\right.} \\
& \left.-\overline{\Delta A} \underline{x}^{+}+\underline{\Delta A^{-}} \underline{x}^{-}\right]+\bar{L} y+|\bar{L}| V E_{p}+\bar{d}(t), \\
& \underline{x}(0)=\underline{x}_{0}, \bar{x}(0)=\bar{x}_{0} .
\end{aligned}
$$

Note that due to the presence of $\underline{x}^{+}, \underline{x}^{-}, \bar{x}^{+}$and $\bar{x}^{-}$, the interval observer (35) is a globally Lipschitz nonlinear system. In addition, in (35) the dynamics of $\underline{x}$ and $\bar{x}$ are coupled. A similar observer has been presented in [56].

Theorem 9. [34] Let assumption 4 be satisfied and the matrices $A_{0}-\underline{L} C, A_{0}-\bar{L} C$ be Metzler. Then the relations (34) hold. If there exist $P \in \mathbb{R}^{2 n \times 2 n}, P=P^{T} \succ 0$ and $\gamma>0$ such that the following Riccati matrix inequality is verified

$$
G^{T} P+P G+2 \gamma^{-2} P^{2}+\gamma^{2} \eta^{2} I_{2 n}+Z^{T} Z \prec 0
$$

where $\eta=2 n\|\overline{\Delta A}-\underline{\Delta A}\|_{\max }, Z \in \mathbb{R}^{s \times 2 n}, 0<s \leq 2 n$ and

$$
G=\left[\begin{array}{cc}
A_{0}-\underline{L} C+\underline{\Delta A^{+}} & -\Delta A^{-} \\
-\overline{\Delta A}^{-} & A_{0}-\bar{L} C+\overline{\Delta A}^{+}
\end{array}\right]
$$

then $\underline{x}, \bar{x} \in \mathcal{L}_{\infty}^{n}$. In addition, the operator $\left[\begin{array}{l}\underline{b} \\ \bar{b}\end{array}\right] \rightarrow Z\left[\begin{array}{l}\underline{x} \\ \bar{x}\end{array}\right]$ in (35) has an $L_{2}$ gain less than $\gamma$.

As in subsection V-B for the interval observer (33), a transformation of coordinates $T$ can also be used for (35) in order to relax the requirement of Theorem 9 that the matrices $A_{0}-\underline{L} C, A_{0}-\bar{L} C$ should be Metzler. 

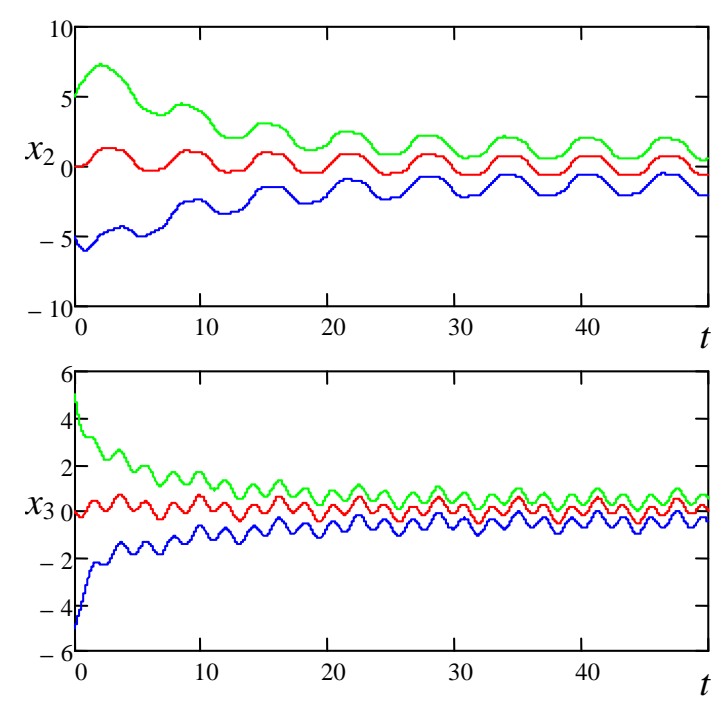

Figure 6. The results of simulations for an LPV system [34]

The Riccati matrix inequality from Theorem 9 can be reformulated in terms of LMIs with respect to $\underline{L}, \bar{L}$ and $P$ [34].

Example 6. Consider a nonlinear system [34]:

$$
\dot{x}=\left[\begin{array}{ccc}
\epsilon \cos t & 1+\epsilon \sin x_{3} & \varepsilon \sin x_{2} \\
\varepsilon \sin x_{3} & -0.5+\epsilon \sin t & 1+\epsilon \cos 2 t \\
\varepsilon \sin x_{2} & 0.3+\varepsilon \cos 2 t & -1+\epsilon \sin t
\end{array}\right] x+\left[\begin{array}{c}
6 \cos x_{1} \\
\sin t+0.1 \sin x_{3} \\
-\cos 3 t+0.1 \sin 2 x_{2}
\end{array}\right], y=x_{1}+v(t),
$$

where $\epsilon=0.01$ and $\varepsilon=0.001$. We assume that $V=0.1$, and for simulation we selected $v(t)=V(\sin 5 t+\cos 3 t) / 2$. For initial conditions $\left|x_{i}(0)\right| \leq 5$ the system has bounded solutions. This system can be presented in the form of (31) for

$$
\begin{gathered}
A_{0}=\left[\begin{array}{ccc}
0 & 1 & 0 \\
0 & -0.5 & 1 \\
0 & 0.3 & -1
\end{array}\right], \overline{\Delta A}=\left[\begin{array}{ccc}
\epsilon & \epsilon & \varepsilon \\
\varepsilon & \epsilon & \epsilon \\
\varepsilon & \varepsilon & \epsilon
\end{array}\right]=-\underline{\Delta A}, \\
\underline{b}(t, y)=\left[\begin{array}{c}
6 \underline{f}(y) \\
\sin t-0.1 \\
-\cos 3 t-0.1
\end{array}\right], \bar{b}(t, y)=\left[\begin{array}{c}
6 \bar{f}(y) \\
\sin t+0.1 \\
-\cos 3 t+0.1
\end{array}\right], \\
\underline{f}(y)=\left\{\begin{array}{ll}
\cos y \cos V & \text { if } \cos y \geq 0 \\
\cos y & \text { if } \cos y<0
\end{array}-|\sin y| \sin V, \bar{f}(y)=\left\{\begin{array}{ll}
\cos y & \text { if } \cos y \geq 0 \\
\cos y \cos V & \text { if } \cos y<0
\end{array}+|\sin y| \sin V\right.\right.
\end{gathered}
$$

and a properly selected $\rho$, clearly assumption 4 is satisfied. Solution of LMIs formulated for Theorem 9 gives the $L_{2}$ optimal solution [34]:

$$
\underline{L}=\left[\begin{array}{c}
82.923 \\
-3 e-4 \\
-4 e-4
\end{array}\right], \bar{L}=\left[\begin{array}{c}
97.16 \\
-2 e-5 \\
-1 e-5
\end{array}\right]
$$

for $\gamma=31.4$ (YALMIP toolbox [57] of MATLAB has been used), where the matrix $Z$ is selecting the variables $x_{2}$ and $x_{3}$. The results of interval simulations for the variables $x_{2}$ and $x_{3}$ are given in Fig. 6 (the variable $x_{1}$ is omitted since it is available for measurements).

Design of observers for discrete-time LPV systems is considered in [58]. Let us consider an example from that paper in comparison with conventional set-membership estimators from [13], [14], [16].

Example 7. There exist many methods to design set-membership estimators [13], [14], [16], i.e. estimators that evaluate 
at each instant of time the set of admissible values for the state, but they have not a structure of observer. Most of setmembership estimation techniques are based on the prediction/correction mechanism. The prediction consists in propagating the state/parameter set available at $t_{k}$ in order to predict an outer approximation at $t_{k+1}$, while the correction step uses the measurement available at $t_{k+1}$ to contract the predicted set. Depending on the system model (linear/nonlinear, continuous/discrete-time), the state set is wrapped into particular geometrical shapes such as ellipsoids [59], zonotopes [60] or intervals [13], [61]. For LTI systems, there exist several efficient solutions to perform state/estimation with guaranteed performances at a low computational cost (for instance by using ellipsoids, zonotopes or polytopes). This is not the case for nonlinear/uncertain systems, where usually interval arithmetic is used. The advantage of interval analysis is that it is possible to propagate uncertainties for nonlinear and LPV systems. Nevertheless, the stability analysis of these algorithms is complicated, in the presence of large uncertainties, due to wrapping and dependence effects (see for instance [62]). In several works, the conservatism is reduced, at the cost of a high complexity, by using sub-pavings and constraint satisfaction techniques. Furthermore, in the case of continuous-time systems, the task is more complicated. Indeed, an uncertain ordinary differential equation (ODE) has to be solved in a validated way at each measurement time instant. Nevertheless, it is well-known that validated integration of ODEs cannot be applied in the case of large uncertainties and the set-membership estimator can diverge even if the considered system is stable. To overcome the conservatism, interval observers, which are detailed in this paper, can be used, where two suitable conventional/pointwise observers are designed in order to estimate, at each time, an outer approximation for the state vector. To illustrate the statements given above, consider a discrete-time system described by

$$
x_{t+1}=\left(A_{0}+\Delta A\right) x_{t}+B u_{t}+d_{t}
$$

with:

$$
\begin{gathered}
A_{0}=\frac{1}{10}\left[\begin{array}{ccc}
0 & 1 & 3 \\
0 & 8 & 2 \\
-0.1 & 0 & 8
\end{array}\right], B=\left[\begin{array}{l}
0 \\
0 \\
1
\end{array}\right], C=\left[\begin{array}{l}
1 \\
0 \\
0
\end{array}\right]^{T}, \\
\overline{\Delta A}=0.015\left[\begin{array}{ccc}
0.1 & 1 & 1 \\
0.1 & 0.1 & 1 \\
0.1 & 0.1 & 1
\end{array}\right], \underline{\Delta A}=-\overline{\Delta A} \\
V=0.2, v_{t}=V \sin (t), \\
d_{t}=\left[\begin{array}{l}
1 \\
1 \\
0
\end{array}\right] \sin (C x)+e_{t},\|e\| \leq \epsilon=0.1 .
\end{gathered}
$$

For simulation we selected

$$
\begin{gathered}
\Delta A\left(\rho_{t}\right)=0.015 \times\left[\begin{array}{ccc}
0.1 \sin \left(\omega_{1} t\right) & \sin \left(\omega_{2} t\right) & \cos \left(\omega_{1} t\right) \\
\cos \left(\omega_{2} t\right) & 0.1 \sin \left(2 \omega_{1} t\right) & \cos \left(2 \omega_{1} t\right) \\
0.1 \sin \left(\omega_{1} t / 2\right) & 0.1 \cos \left(\omega_{2} t / 2\right) & \sin \left(\omega_{1} t\right) \cos \left(\omega_{2} t\right)
\end{array}\right], \\
e_{t}=\epsilon\left[\begin{array}{c}
\sin \left(\omega_{2} t\right) \\
\sin \left(\omega_{1} t\right) \\
\cos \left(\omega_{2} t\right)
\end{array}\right], \omega_{1}=0.2, \omega_{2}=1 / 3
\end{gathered}
$$

Consider the interval observer proposed in [58] with the gains

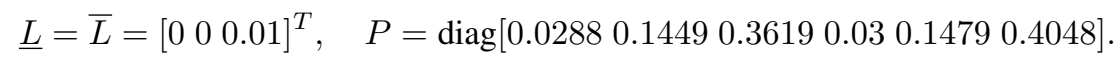

For this numerical example, the results of simulations with the interval observer of [58] and those computed by the prediction/correction estimator are plotted in Fig 7. The simulations show clearly that the prediction/correction estimator generates more conservative results (larger intervals). Instead of outer approximating the feasible state set, subpavings (union of intervals) are used in [62] which is a time-consuming procedure. 

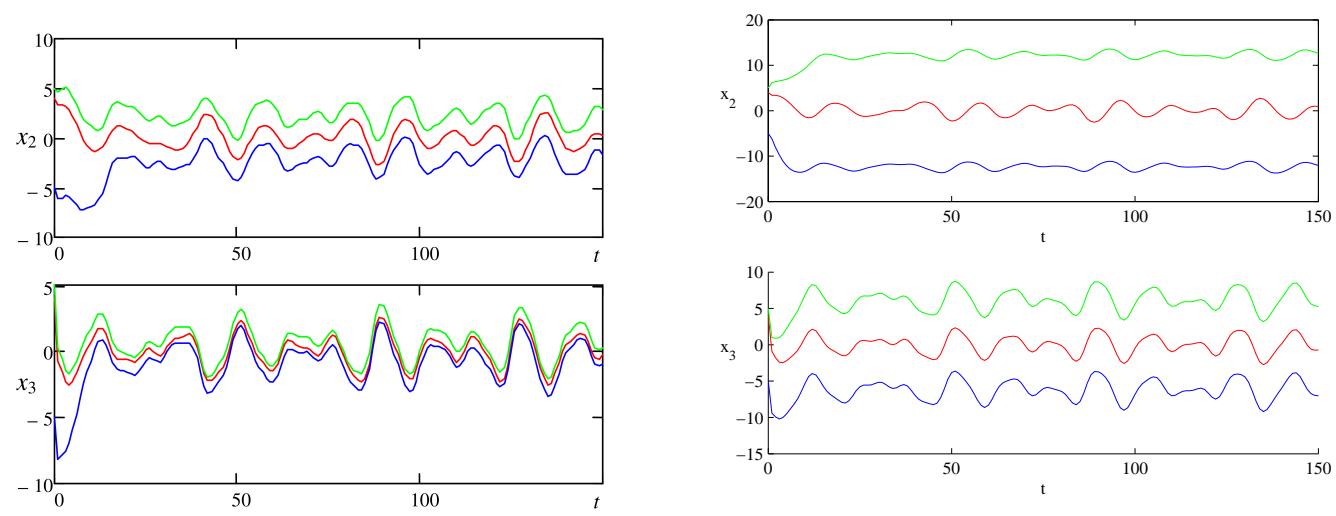

Figure 7. The results of simulations: interval observer on the left; prediction/correction on the right [58]

\section{The case of LTV systems}

LTV models can be considered as a special case of LPV ones when the vector of scheduling parameters is available for measurements. Interval observers have been proposed for LTV systems in [54], [63], where different variants of transformations of time-varying systems to nonnegative forms are presented. Consider a LTV system described by:

$$
\begin{aligned}
& \dot{x}(t)=A(t) x(t)+d(t), \\
& y(t)=C(t) x(t)+v(t), \\
& x\left(t_{0}\right) \in \mathbb{R}^{n}, t \geq t_{0} \geq 0,
\end{aligned}
$$

where $x(t) \in \mathbb{R}^{n}, d(t) \in \mathbb{R}^{n}, y(t) \in \mathbb{R}^{p}$ and $v(t) \in \mathbb{R}^{p}$ are respectively the state vector, an unknown but bounded input, the output vector and a bounded noise.

Assumption 6. Let $x\left(t_{0}\right) \in\left[\underline{x}_{0}, \bar{x}_{0}\right]$ for some known $\underline{x}_{0}, \bar{x}_{0} \in \mathbb{R}^{n} ; d(t) \in[\underline{d}(t), \bar{d}(t)]$ for all $t \geq t_{0}$, where $\underline{d}, \bar{d} \in \mathcal{L}_{\infty}^{n}$; $v(t) \in[\underline{v}(t), \bar{v}(t)]$ for all $t \geq t_{0}$, where $\underline{v}, \bar{v} \in \mathcal{L}_{\infty}^{p}$.

So, as before, the model uncertainty is represented by intervals of initial conditions, state disturbances $d(t)$ and measurement noises $v(t)$. In [63], an effective technique has been proposed to build an interval observer for systems described by (36).

Assumption 7. There exist bounded matrix functions $L: \mathbb{R} \rightarrow \mathbb{R}^{n \times p}, M: \mathbb{R}_{+} \rightarrow \mathbb{R}^{n \times n}, M(\cdot)=M(\cdot)^{T} \succ 0$ such that for all $t \geq t_{0}$,

$$
\dot{M}(t)+D(t)^{T} M(t)+M(t) D(t) \prec 0, \quad D(t)=A(t)-L(t) C(t) .
$$

Assumption 7 is a conventional requirement for LTV systems [64]. Under this assumption, the observer gain $L(t)$ and the matrix function $M(t)$ are such that the stability of the LTV system $\dot{x}(t)=D(t) x(t)$ can be proven by taking $V(t)=$ $x(t)^{T} M(t) x(t)$ as a Lyapunov function. It determines the output stabilization conditions of the system (36) which can be rewritten as:

$$
\begin{aligned}
\dot{x}(t) & =D(t) x(t)+\tilde{d}(t), \\
y(t) & =C(t) x(t)+v(t)
\end{aligned}
$$

with $\tilde{d}(t)=d(t)-L(t) v(t)+L(t) y(t)$.

When the gain $L(t)$ is computed such that the closed loop matrix $D(t)$ is stable and Metzler, the observer design is similar to the ones for LTI and LPV systems. In [63], such a restrictive condition has been avoided. The methodology is based on the D-similarities approach developed in [65], [66], [67], where any time-varying matrix can be transformed under a Metzler form. 
Proposition 2. There exists a time-varying transformation $z=T(t) x$ transforming $D(t)$ into a Metzler matrix $\Gamma(t)$ :

$$
\Gamma(t)=T(t)\left(D(t) T^{-1}(t)-d\left(T^{-1}(t)\right) / d t\right)
$$

where $T(t)=P(t) L^{-1}(t)$ with

$$
\Gamma(t)=\left[\begin{array}{cccc}
\lambda_{1}(t) & 1 & \cdots & 0 \\
0 & \lambda_{2}(t) & \ddots & \vdots \\
\vdots & \ddots & \ddots & 1 \\
0 & \cdots & 0 & \lambda_{n}(t)
\end{array}\right]
$$

The elements $\lambda_{i}(t)$ are called Essential D-eigenvalues of $D(t)$ (or ED-eigenvalues, see Definition 3.1 in [65]).

Now, design of an interval observer requires the following classical assumption.

Theorem 10. [63] Let assumptions 6 and 7 be satisfied for (36) and $\|y(t)\| \leq Y$ for all $t \geq t_{0}$ for a known constant $Y>0$. Given the matrix $T$ defined in Proposition 2, assume that $\exists M_{1} \in \mathbb{R}_{+}$such that $\|T(t)\|+\left\|T^{-1}(t)\right\| \leq M_{1}$ for all $t \geq t_{0}$. Then,

$$
\begin{aligned}
& \dot{\dot{z}}(t)=\Gamma(t) \underline{z}(t)+\underline{d}_{o b s}(t)+\underline{\Psi}_{o b s}(t)+T_{o b s}(t) y(t) \\
& \dot{\bar{z}}(t)=\Gamma(t) \bar{z}(t)+\bar{d}_{o b s}(t)+\bar{\Psi}_{o b s}(t)+T_{o b s}(t) y(t)
\end{aligned}
$$

is an interval observer for (37) and

$$
\underline{z}(t) \leq T(t) x(t) \leq \bar{z}(t), \forall t \geq t_{0}
$$

where $\underline{d}_{o b s}(t)=T^{+}(t) \underline{d}(t)-T^{-}(t) \bar{d}(t), T_{o b s}(t)=T(t) L(t), \underline{\Psi}_{o b s}(t)=T_{o b s}^{-}(t) \underline{v}(t)-T_{o b s}^{+}(t) \bar{v}(t), \bar{d}$ obs $(t)=T^{+}(t) \bar{d}(t)-$ $T^{-}(t) \underline{d}(t), \bar{\Psi}_{o b s}(t)=T_{o b s}^{-}(t) \bar{v}(t)-T_{o b s}^{+}(t) \underline{v}(t)$.

\section{APPLICATION OF INTERVAL OBSERVERS FOR CONTROL}

Recently, interval observers have been used in several works for control design of both continuous and discrete-time systems [68], [69], [70], [58], [71]. In the following, the methodology is shown only for LPV systems described by (31). Nevertheless, the reader can refer for instance to [58], [71] for nonlinear and discrete-time models.

The considered previously models (7), (14) and (31) do not contain control, it can be easily introduced, as in (31) for example:

$$
\dot{x}=\left[A_{0}+\Delta A(\rho(t))\right] x+B(\rho(t)) u+d(t), y=C x+v(t), t \geq 0,
$$

where $u \in \mathbb{R}^{m}$ is the control input and $B: \Pi \rightarrow \mathbb{R}^{n \times m}$ is a piecewise continuous matrix function that known for a given value of $\rho$. Then there exist matrices $B_{\min }, B_{\max }$ such that the relations

$$
B_{\min } \leq B(\rho) \leq B_{\max }
$$

are satisfied provided that $\underline{x} \leq x \leq \bar{x}$ and $\rho \in \Pi$, and for any $u \in \mathbb{R}^{m}$ the inequalities

$$
\underline{B}(u) u \leq B(\theta) u \leq \bar{B}(u) u
$$

are valid for

$$
\underline{B}^{(i)}(u)=\left\{\begin{array}{l}
B_{\min }^{(i)} \text { if } u_{i} \geq 0 ; \\
B_{\max }^{(i)} \text { if } u_{i}<0,
\end{array} \quad \bar{B}^{(i)}(u)=\left\{\begin{array}{c}
B_{\max }^{(i)} \text { if } u_{i} \geq 0 \\
B_{\min }^{(i)} \text { if } u_{i}<0
\end{array}\right.\right.
$$

where the upper index $i$ for a matrix $B^{(i)}$ denotes the $i$-th column of the matrix, and the lower index $i$ for a vector $u_{i}$ returns 
the $i$-th element of the vector. In this case the interval observer (35) admits a mild modification:

$$
\begin{aligned}
\underline{\dot{x}}= & {\left[A_{0}-\underline{L} C\right] \underline{x}+\left[\underline{\Delta A}^{+} \underline{x}^{+}-\overline{\Delta A}^{+} \underline{x}^{-}\right.} \\
& \left.-\Delta A^{-} \bar{x}^{+}+\overline{\Delta A} \bar{x}^{-}\right]+\underline{B}(u) u+\underline{L} y-|\underline{L}| E_{p} V+\underline{d}(t), \\
\dot{\bar{x}}= & {\left[A_{0}-\bar{L} C\right] \bar{x}+\left[\overline{\Delta A} \bar{x}^{+}-\underline{\Delta A^{+}} \bar{x}^{-}\right.} \\
& \left.-\overline{\Delta A} \underline{x}^{+}+\underline{\Delta A^{-}} \underline{x}^{-}\right]+\bar{B}(u) u+\bar{L} y+|\bar{L}| E_{p} V+\bar{d}(t), \\
& \underline{x}(0)=\underline{x}_{0}, \bar{x}(0)=\bar{x}_{0},
\end{aligned}
$$

and the proof that the interval inclusion (34) is satisfied under conditions of Theorem 9 is the same as in the above theorem.

The main idea of control design using interval observers is as follows [69]. From (34), if both $\underline{x}(t)$ and $\bar{x}(t)$ converge to zero, then the state $x(t)$ also has to converge to zero, and boundedness of $x(t)$ follows by the same property of $\underline{x}(t)$ and $\bar{x}(t)$. Thus, it is necessary to design a control $u=u(\underline{x}, \bar{x}, y)$ stabilizing the observer $(41)$. In this case the signal $y(t)$ is treated in the system (40) as a state dependent disturbance with an upper bound

$$
|y(t)| \leq|C|(|\underline{x}(t)|+|\bar{x}(t)|), \forall t \geq 0 \text {. }
$$

Therefore, it is required to stabilize the system (41) uniformly (or robustly) with respect to the input $y$. The advantages of such a reduction are that the system (41) is completely known and the state vector $\underline{x}(t), \bar{x}(t)$ is available for design. However, the dimension of (41) is twice bigger than the corresponding dimension of the system (40) while the control vector $u$ preserves its size. The control can be chosen as a conventional state linear feedback:

$$
u=\underline{K x}+\bar{K} \bar{x}
$$

where $\underline{K} \in \mathbb{R}^{m \times n}$ and $\bar{K} \in \mathbb{R}^{m \times n}$ are two feedback gains to be designed. All the gains $\underline{L}, \bar{L}, \underline{K}$ and $\bar{K}$ can be used to ensure the closed-loop system stability (nonnegativity of the estimation error dynamics has to be ensured by a proper choice of $\underline{L}$ and $\bar{L}$ ), thus the restrictions on $\underline{L}, \bar{L}$ are relaxed (stability can be provided by the feedback).

To simplify notation consider the case $B(\rho)=B$ and $\underline{\Delta A}=-\overline{\Delta A}, \overline{\Delta A} \geq 0$, then (41) with the control takes the form [69]:

$$
\begin{aligned}
\underline{\dot{x}}= & {\left[A_{0}-\underline{L} C+B \underline{K}\right] \underline{x}+B \bar{K} \bar{x}-\overline{\Delta A}\left[\bar{x}^{+}+\underline{x}^{-}\right]+\underline{L} y+\underline{d}(t), } \\
\dot{\bar{x}}= & {\left[A_{0}-\bar{L} C+B \bar{K}\right] \bar{x}+B \underline{K x}+\overline{\Delta A}\left[\bar{x}^{+}+\underline{x}^{-}\right]+\bar{L} y+|\bar{L}| E_{p} V+\bar{d}(t), } \\
& \underline{x}(0)=\underline{x}_{0}, \bar{x}(0)=\bar{x}_{0} .
\end{aligned}
$$

Denote $\xi=\left[\underline{x}^{\mathrm{T}} \bar{x}^{\mathrm{T}}\right]^{\mathrm{T}}$,

$$
G=\left[\begin{array}{cc}
A_{0}-\underline{L} C+B \underline{K} & B \bar{K} \\
B \underline{K} & A_{0}-\bar{L} C+B \bar{K}
\end{array}\right], f(\xi, y)=\left[\begin{array}{c}
\underline{L} y-\overline{\Delta A}\left[\bar{x}^{+}+\underline{x}^{-}\right] \\
\bar{L} y+\overline{\Delta A}\left[\bar{x}^{+}+\underline{x}^{-}\right]
\end{array}\right],
$$

then

$$
\dot{\xi}=G \xi+f(\xi, y)+\left[\begin{array}{l}
\underline{d}(t)-|\underline{L}| E_{p} V \\
\bar{d}(t)+|\bar{L}| E_{p} V
\end{array}\right]
$$

where $|f(\xi, y)| \leq \gamma|\xi|$ for $\gamma=\max \left\{\|\underline{L}\|_{2},\|\bar{L}\|_{2}\right\}\|C\|_{2}+\sqrt{2}\|\overline{\Delta A}\|_{2}$.

Theorem 11. Let matrices $A_{0}-\underline{L} C, A_{0}-\bar{L} C$ be Metzler, then the relations (34) are satisfied for (40) and (41). In addition, if there exist matrix $P^{T}=P \succ 0$ such that

$$
\left[\begin{array}{cc}
G^{T} P+P G+\gamma^{2} I_{n} & P \\
P & -I_{n}
\end{array}\right] \prec 0
$$

then the system (40), (41), (42) has bounded solutions.

The above LMI can be rewritten in a way to find also the control gains $\underline{K}$ and $\bar{K}$ [69]. 

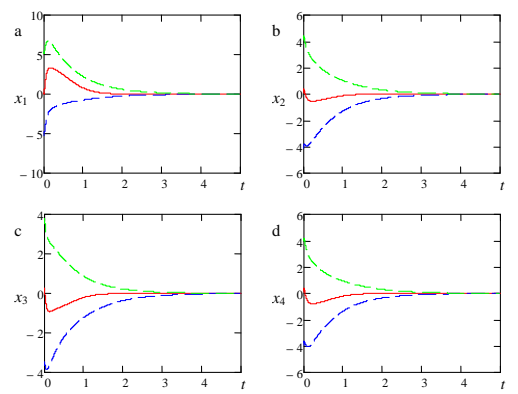

Figure 8. The state trajectories of LPV system [69]

Example 8. Consider an uncertain system:

$$
\begin{gathered}
\dot{x}=A(\theta) x+B u, y=x_{1}, \\
A-\Delta A \leq A(\theta) \leq A+\Delta A, \theta \in \mathbb{R}, \\
A=\left[\begin{array}{cccc}
35.6 & 50.7 & 45.6 & 75.6 \\
-1.8 & -25.5 & -3.8 & -6.3 \\
-18.1 & -20 & -38.9 & -31 \\
-5.8 & -7 & -6.6 & -30.5
\end{array}\right], \\
\Delta A=\frac{1}{4}\left[\begin{array}{cccc}
1 & 1 & 5 & 2 \\
1 & 2 & 1 & 3 \\
2 & 1 & 2 & 2 \\
1 & 4 & 1 & 2
\end{array}\right], B=\left[\begin{array}{cc}
1 & -1 \\
2 & 3 \\
-2 & 1 \\
-2 & 2
\end{array}\right],
\end{gathered}
$$

where the matrix $\Delta A$ defines the admissible time-varying deviations from the nominal value $A$. For unstable matrix $A$ there is no $L$ such that $A-L C$ is Metzler, however for

$$
L=\left[\begin{array}{llll}
5 & 2 & 3 & 1
\end{array}\right]^{T}, T=\left[\begin{array}{cccc}
-22.179 & 8 & 3 & 19 \\
8 & -21.179 & 7 & 5 \\
3 & 7 & -20.179 & 6 \\
9 & 5 & 6 & -19.179
\end{array}\right]
$$

the matrix $T^{-1}(A-L C) T$ is Hurwitz and Metzler as it is required. In addition, all other conditions of Theorem 11 are satisfied for [69]:

$$
\begin{gathered}
\underline{K}=\left[\begin{array}{cccc}
-50.76 & -50.92 & -50.59 & -50.72 \\
267.17 & 267.91 & 266.30 & 26.08
\end{array}\right], \\
\bar{K}=\left[\begin{array}{cccc}
152.56 & 151.80 & 152.05 & 151.83 \\
-799.02 & -795.32 & -796.08 & -796.11
\end{array}\right],
\end{gathered}
$$

For simulation we choose $A[\theta(t)]=A(t)=A+V(t)$, where

$$
V(t)=\frac{1}{4}\left[\begin{array}{cccc}
\sin (t) & \cos (0.5 t) & 5 \sin (2 t) & 2 \cos (t) \\
\sin (0.5 t) & 2 \cos (2 t) & \cos (t) & 3 \sin (0.5 t) \\
2 \cos (2 t) & \sin (t) & 2 \cos (0.5 t) & 2 \sin (2 t) \\
\cos (t) & 4 \sin (0.5 t) & \cos (2 t) & 2 \sin (0.5 t)
\end{array}\right] .
$$

The results of simulation are presented in figures 8 and 9. On plots Fig. 8,a - Fig. 8,d the state coordinates are shown (solid line) with the corresponding bounding variables from the interval observer (dashed lines). In Fig. 9 the controls amplitudes are given in the logarithmic scale.

Control of discrete-time LPV systems is studied in [58], [71] (in [71] the control is dependent only on lower $\underline{x}$ or upper $\bar{x}$ 


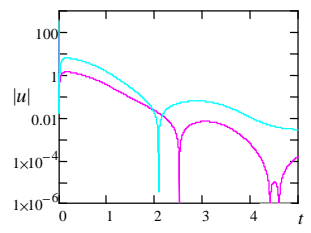

Figure 9. The control amplitudes for LPV system [69]

estimate).

\section{CONCLUSION AND OPEN PROBLEMS}

In the present survey the main approaches to design interval observers are introduced. Interval observers are conceptually different from standard pointwise observers, and the main advantage of an interval observer is that it also guarantees estimation error values in the calculated bounds. The given survey is not complete and there are many other important works in the domain of design of interval observers, which are not mentioned here. Some of them are briefly mentioned below.

Recursive design of ISS interval observers for lower-triangular systems is considered in [72]. An interesting result on application of interval observers for stability analysis is given in [73], where for a nonlinear time-varying delayed system an interval observer is proposed enveloping the original system solutions, then stability of the observer implies stability of the system (similarly as control has been designed above in [69]).

Sliding mode control and estimation algorithms are well known for they compensation of matched disturbances and finite-time convergence. Combination of sliding mode differentiators (used for decreasing the system uncertainty) and interval observers is reported in [20].

The interval observers and set-membership estimation techniques are effectively used for fault detection and isolation in different applications [74], [75], [76]. Another important and traditional application for interval observers is state estimation in biological systems [21], [15], [17]. Applications of interval observers in automotive domain are considered in [77], [78] (the problem of air-to-fuel ratio interval estimation and control is studied in [77] and a car positioning problem is solved in [78] using interval tools).

Despite the fact that interval observers have been already developed for many classes of systems, still there exist a lot of open problems, mainly for time-delay and distributed (partial differential equations) models, differential inclusions, hybrid and switched systems. Another generic direction of development deals with constructive tools for observer construction (LMI or LP formulations [33]) and development of necessary and sufficient conditions of existence of interval observers, optimization of interval accuracy is also very important in that way. Interval observers confirmed their ability for guaranteed interval estimation in uncertain systems, expanding the areas of application of interval observers is another open direction of research.

\section{REFERENCES}

[1] T. Meurer, K. Graichen, and E.-D. Gilles, eds., Control and Observer Design for Nonlinear Finite and Infinite Dimensional Systems, vol. 322 of Lecture Notes in Control and Information Sciences. Springer, 2005.

[2] T. Fossen and H. Nijmeijer, New Directions in Nonlinear Observer Design. Springer, 1999.

[3] G. Besançon, ed., Nonlinear Observers and Applications, vol. 363 of Lecture Notes in Control and Information Sciences. Springer, 2007.

[4] F. Esfandiari and H. Khalil, "Output feedback stabilization of fully linearizable systems," Int. J. Control, vol. 56, pp. 1007-1037, 1992.

[5] A. Levant, "High-order sliding modes: differentiation and output feedback control," Int. J. Control, vol. 76, no. 9-10, pp. 924-941, 2003.

[6] A. Golubev, A. Krishchenko, and S. Tkachev, "Stabilization of nonlinear dynamic systems using the system state estimates made by the asymptotic observer," Automation and Remote Control, vol. 66, no. 7, pp. 1021-1058, 2005

[7] J. Shamma, Control of Linear Parameter Varying Systems with Applications, ch. An overview of LPV systems, pp. 1-22. Springer, 2012.

[8] A. Marcos and J. Balas, "Development of linear parameter-varying models for aircraft," J. Guidance, Control, Dynamics, vol. 27, no. 2, pp. 218-228, 2004.

[9] J. Shamma and J. Cloutier, "Gain-scheduled missile autopilot design using linear parameter-varying transformations," J. Guidance, Control, Dynamics, vol. 16, no. 2, pp. 256-261, 1993.

[10] W. Tan, Applications of Linear Parameter-Varying Control Theory. PhD thesis, Dept. of Mechanical Engineering, University of California at Berkeley, 1997.

[11] Y. Shtessel, C. Edwards, L. Fridman, and A. Levant, Sliding Mode Control and Observation. Control Engineering, Birkhäuser Basel, 2013. 
[12] C. Guanrong, W. Jianrong, and S. Leang, "Interval kalman filtering," IEEE Transactions on Aerospace and Electronic Systems, vol. 33, no. 1, pp. 250-259, 1997.

[13] L. Jaulin, "Nonlinear bounded-error state estimation of continuous time systems," Automatica, vol. 38, no. 2, pp. 1079-1082, 2002.

[14] M. Kieffer and E. Walter, "Guaranteed nonlinear state estimator for cooperative systems," Numerical Algorithms, vol. 37, pp. 187-198, 2004.

[15] B. Olivier and J. Gouzé, "Closed loop observers bundle for uncertain biotechnological models," Journal of Process Control, vol. 14, no. 7, pp. 765-774, 2004.

[16] M. Milanese and C. Novara, "Unified set membership theory for identification, prediction and filtering of nonlinear systems," Automatica, vol. 47, no. 10, pp. 2141-2151, 2011.

[17] M. Moisan, O. Bernard, and J. Gouzé, "Near optimal interval observers bundle for uncertain bio-reactors," Automatica, vol. 45, no. 1, pp. 291-295, 2009.

[18] T. Raïssi, G. Videau, and A. Zolghadri, "Interval observers design for consistency checks of nonlinear continuous-time systems," Automatica, vol. 46, no. 3, pp. 518-527, 2010.

[19] T. Raïssi, D. Efimov, and A. Zolghadri, "Interval state estimation for a class of nonlinear systems," IEEE Trans. Automatic Control, vol. 57, no. 1, pp. 260-265, 2012.

[20] D. Efimov, L. Fridman, T. Raïssi, A. Zolghadri, and R. Seydou, "Interval estimation for LPV systems applying high order sliding mode techniques," Automatica, vol. 48, pp. 2365-2371, 2012.

[21] J. Gouzé, A. Rapaport, and M. Hadj-Sadok, "Interval observers for uncertain biological systems," Ecological Modelling, vol. 133, pp. 46-56, 2000.

[22] F. Mazenc and O. Bernard, "Interval observers for linear time-invariant systems with disturbances," Automatica, vol. 47, no. 1, pp. 140-147, 2011.

[23] C. Combastel, "Stable interval observers in C for linear systems with time-varying input bounds," Automatic Control, IEEE Transactions on, vol. 58, no. 2, pp. 481-487, 2013.

[24] L. Farina and S. Rinaldi, Positive Linear Systems: Theory and Applications. New York: Wiley, 2000.

[25] H. Smith, Monotone Dynamical Systems: An Introduction to the Theory of Competitive and Cooperative Systems, vol. 41 of Surveys and Monographs. Providence: AMS, 1995.

[26] C. Briat, "Robust stability analysis of uncertain linear positive systems via integral linear constraints: $l_{1}-$ and $l_{\infty}$-gain characterizations," in Proc. 50th IEEE CDC and ECC, (Orlando), pp. 6337-6342, 2011.

[27] Y. Ebihara, D. Peaucelle, and D. Arzelier, "L1 gain analysis of linear positive systems and its application," in Proc. 50th IEEE CDC and ECC, (Orlando), pp. 4029-4035, 2011.

[28] H. K. Khalil, Nonlinear Systems. Prentice Hall PTR, 3rd ed., 2002.

[29] M. W. Hirsch and H. L. Smith, "Monotone maps: a review," J. Difference Equ. Appl., vol. 11, no. 4-5, pp. 379-398, 2005.

[30] W. Haddad and V. Chellaboina, "Stability theory for nonnegative and compartmental dynamical systems with time delay," Syst. Control Letters, vol. 51, pp. 355-361, 2004.

[31] M. Dambrine and J.-P. Richard, "Stability analysis of time-delay systems," Dynamic Systems and Applications, vol. 2, pp. 405-414, 1993.

[32] D. Efimov, A. Polyakov, E. M. Fridman, W. Perruquetti, and J.-P. Richard, "Delay-dependent positivity: Application to interval observers," in Proc. ECC 2015, (Linz), 2015.

[33] P. McCarthy, C. Nielsen, and S. Smith, "Cardinality constrained robust optimization applied to a class of interval observers," in American Control Conference (ACC), 2014, pp. 5337-5342, 2014.

[34] S. Chebotarev, D. Efimov, T. Raïssi, and A. Zolghadri, "Interval observers for continuous-time LPV systems with $l_{1} / l_{2}$ performance," Automatica, vol. 58, no. 8, pp. 82-89, 2015.

[35] F. Cacace, A. Germani, and C. Manes, "A new approach to design interval observers for linear systems," IEEE Trans. Automatic Control, vol. 99, no. 99, pp. 1-1, 2015.

[36] F. Mazenc and O. Bernard, "Asymptotically stable interval observers for planar systems with complex poles," IEEE Transactions on Automatic Control, vol. 55, no. 2, pp. 523-527, 2010.

[37] D. Efimov, W. Perruquetti, T. Raïssi, and A. Zolghadri, "On interval observer design for time-invariant discrete-time systems," in Proc. European Control Conference (ECC) 2013, (Zurich), 2013.

[38] F. Mazenc, T. N. Dinh, and S. I. Niculescu, "Interval observers for discrete-time systems," in 51th IEEE Conference on Decision and Control, (Hawaii), pp. 6755-6760, 2012.

[39] F. Mazenc, T. N. Dinh, and S. I. Niculescu, "Interval observers for discrete-time systems," International Journal of Robust and Nonlinear Control, vol. 24, pp. 2867-2890, 2014.

[40] L. Farina and S. Rinaldi, Positive Linear Systems - Theory and Applications. NY: John Wiley \& Sons, 2000.

[41] D. Efimov, W. Perruquetti, T. Raïssi, and A. Zolghadri, "Interval observers for time-varying discrete-time systems," IEEE Trans. Automatic Control, vol. 58, no. 12 , pp. 3218-3224, 2013.

[42] D. Efimov, W. Perruquetti, and J.-P. Richard, "Interval estimation for uncertain systems with time-varying delays," International Journal of Control, vol. 86, no. 10, pp. 1777-1787, 2013.

[43] D. Efimov, W. Perruquetti, and J.-P. Richard, “On reduced-order interval observers for time-delay systems,” in Proc. European Control Conference (ECC) 2013, (Zurich), 2013.

[44] V. Kolmanovskii and A. Myshkis, Introduction to the Theory and Applications of Functional Differential Equations. Dordrecht: Kluwer Academic Publishers, 1999.

[45] P. Pepe and Z.-P. Jiang, "A Lyapunov-Krasovskii methodology for ISS and iISS of time-delay systems," Systems \& Control Letters, vol. 55, pp. 10061014,2006

[46] M. Rami, U. Helmke, and F. Tadeo, "Positive observation problem for linear time-delay positive systems," in Proc. Mediterranean Conf. Control \& Automation (MED '07), pp. 1-6, 2007. 
[47] F. Mazenc, S. I. Niculescu, and O. Bernard, "Exponentially stable interval observers for linear systems with delay," SIAM J. Control Optim., vol. 50, pp. 286-305, 2012.

[48] D. Efimov, A. Polyakov, and J.-P. Richard, "Interval observer design for estimation and control of time-delay descriptor systems," European Journal of Control, vol. 23, no. 5, pp. 26-35, 2015.

[49] A. Churilov, A. Medvedev, and A. Shepeljavyi, "Mathematical model of non-basal testosterone regulation in the male by pulse modulated feedback," Automatica, vol. 45, pp. 78-85, 2009.

[50] D. Greenhalgh and Q. Khan, "A delay differential equation mathematical model for the control of the hormonal system of the hypothalamus, the pituitary and the testis in man," Nonlinear Analysis, vol. 71, pp. e925-e935, 2009.

[51] M. Bolajraf, M. Ait Rami, and U. R. Helmke, "Robust positive interval observers for uncertain positive systems," in Proc. of the 18th IFAC World Congress, pp. 14330-14334, 2011.

[52] K. Chen, L. Goh, G. He, V. Bhamidi, P. Kenis, C. Zukoski, and B. R.D., "Identification of nucleation rates in droplet-based microfluidic systems," Chem. Eng. Sci., vol. 77, pp. 235-241, 2012.

[53] L. Goh, K. Chen, V. Bhamidi, G. He, N. C. S. Kee, P. J. A. Kenis, C. F. Zukoski, and R. D. Braatz, "A stochastic model for nucleation kinetics determination in droplet-based microfluidic systems," Crystal Growth \& Design, vol. 10, no. 6, pp. 2515-2521, 2010.

[54] D. Efimov, T. Raïssi, S. Chebotarev, and A. Zolghadri, "Interval state observer for nonlinear time varying systems," Automatica, vol. 49, no. 1, pp. 200-205, 2013.

[55] J. Back and A. Astolfi, "Design of positive linear observers for positive linear systems via coordinate transformations and positive realizations," SIAM J. Control Optim., vol. 47, no. 1, pp. 345-373, 2008.

[56] M. Ait Rami, C. Cheng, and C. de Prada, "Tight robust interval observers: an LP approach," in Proc. of 47th IEEE Conference on Decision and Control, (Cancun, Mexico), pp. 2967-2972, Dec. 9-11 2008.

[57] J. Löfberg, "Automatic robust convex programming," Optimization methods and software, vol. 27, no. 1, pp. 115-129, 2012.

[58] D. Efimov, T. Raïssi, W. Perruquetti, and A. Zolghadri, "Estimation and control of discrete-time LPV systems using interval observers," in Proc. 52nd IEEE Conference on Decision and Control 2013, (Florence), 2013.

[59] B. T. Polyak, S. A. Nazin, C. Durieu, and E. Walter, "Ellipsoidal parameter or state estimation under model uncertainty," Automatica, vol. 40, no. 7, pp. 1171-1179, 2004.

[60] T. Alamo, J. Bravo, and E. Camacho, "Guaranteed state estimation by zonotopes," Automatica, vol. 41, no. 6, pp. 1035-1043, 2005.

[61] T. Raïssi, N. Ramdani, and Y. Candau, "Set membership state and parametre estimation for systems described by nonlilear differential equations," Automatica, vol. 40, pp. 1771-1777, 2004

[62] L. Jaulin, M. Kieffer, O. Didrit, and E. Walter, Applied interval analysis. London: Springer, 2001.

[63] R. E. H. Thabet, T. Raïssi, C. Combastel, D. Efimov, and A. Zolghadri, "An effective method to interval observer design for time-varying systems," Automatica, vol. 50, no. 10, pp. 2677-2684, 2014.

[64] F. Amato, A. Pironti, and S. Scala, "Necessary and sufficient conditions for quadratic stability and stabilizability of uncertain linear time-varying systems," IEEE Transaction On Automatic Control, vol. 41, pp. 125-128, 1996.

[65] J. Zhu and C. D. Johnson, "Unified canonical forms for linear time-varying dynamical systems under D-similarity transformations. Part I," Southeastern Symposium on System Theory, pp. 74-81, 1989.

[66] J. Zhu and C. D. Johnson, “Unified canonical forms for linear time-varying dynamical systems under D-similarity transformations. Part II," Southeastern Symposium on System Theory, pp. 57-63, 1989.

[67] J. Zhu and C. Johnson, “Unified canonical forms for matrices over a differential ring," Linear Algebra and its Applications, vol. 147, no. 0, pp. 201-248, 1991.

[68] D. Efimov, T. Raïssi, and A. Zolghadri, "Stabilization of nonlinear uncertain systems based on interval observers," in Proc. 50th IEEE CDC-ECC 2011, (Orlando, FL), pp. 8157-8162, 2011.

[69] D. Efimov, T. Raïssi, and A. Zolghadri, "Control of nonlinear and lpv systems: interval observer-based framework," IEEE Trans. Automatic Control, vol. 58, no. 3, pp. 773-782, 2013.

[70] X. Cai, G. Lv, and W. Zhang, "Stabilisation for a class of non-linear uncertain systems based on interval observers," Control Theory Applications, IET, vol. 6, no. 13, pp. 2057-2062, 2012.

[71] F. Mazenc, T. N. Dinh, and S. I. Niculescu, "Robust interval observers and stabilization design for discrete-time systems with input and output," Automatica, vol. 49, pp. 3490-3497, 2013.

[72] F. Mazenc and O. Bernard, "ISS interval observers for nonlinear systems transformed into triangular systems," International Journal of Robust and Nonlinear Control, vol. 24, no. 7, pp. 1241-1261, 2014.

[73] F. Mazenc and M. Malisoff, "New technique for stability analysis for time-varying systems with delay," in Proc. 53th IEEE Conference on Decision and Control, (Los Angeles), pp. 1215-1220, 2014.

[74] V. Puig, A. Stancu, T. Escobet, F. Nejjari, J. Quevedo, and R. Patton, "Passive robust fault detection using interval observers: Application to the DAMADICS benchmark problem," Control Engineering Practice, vol. 14, pp. 621-633, 2006.

[75] J. Blesa, V. Puig, and Y. Bolea, "Fault detection using interval LPV models in an open-flow canal," Control Engineering Practice, vol. 18, no. 5, pp. $460-470,2010$.

[76] J. Blesa, D. Rotondo, V. Puig, and F. Nejjari, "FDI and FTC of wind turbines using the interval observer approach and virtual actuators/sensors," Control Engineering Practice, vol. 24, pp. 138-155, 2014

[77] D. Efimov, S. Li, Y. Hu, S. Muldoon, H. Javaherian, and V. Nikiforov, "Application of interval observers to estimation and control of air-fuel ratio in a direct injection engine," in Proc. ACC 2015, (Chicago), 2015.

[78] G. Goffaux, M. Remy, and A. V. Wouwer, "Continuous-discrete confidence interval observer - application to vehicle positioning," Information Fusion, vol. 14, no. 4, pp. 541-550, 2013. 